\title{
On mass transport in porosity waves
}

\author{
Jacob S. Jordan ${ }^{\mathrm{a}, \mathrm{d}, 1}$, Marc A. Hesse ${ }^{\mathrm{a}, \mathrm{b}, 2}$, John F. Rudge ${ }^{\mathrm{c}}$ \\ ${ }^{a}$ The University of Texas at Austin, Department of Geological Sciences, 2305 Speedway \\ Stop C1160, Austin, TX 78712-1692 \\ ${ }^{b}$ The University of Texas at Austin, Institute for Computational Engineering and Sciences, \\ 201 E 24th Street, Stop 0200, Austin, TX 78712-1229 \\ ${ }^{c}$ Bullard Laboratories, Department of Earth Sciences, University of Cambridge, Madingley \\ Road, Cambridge, UK CB3 OEZ \\ ${ }^{d}$ Yale University, Department of Geology and Geophysics, 210 Whitney Avenue, New \\ Haven, CT 06511
}

\begin{abstract}
Porosity waves arise naturally from the equations describing fluid migration in ductile rocks. Here, we show that higher-dimensional porosity waves can transport mass and therefore preserve geochemical signatures, at least partially. Fluid focusing into these high porosity waves leads to recirculation in their center. This recirculating fluid is separated from the background flow field by a circular dividing streamline and transported with the phase velocity of the porosity wave. Unlike models for one-dimensional chromatography in geological porous media, tracer transport in higher-dimensional porosity waves does not produce chromatographic separations between relatively incompatible elements due to the circular flow pattern. This may allow melt that originated from the partial melting of fertile heterogeneities or fluid produced during metamorphism to retain distinct geochemical signatures as they rise buoyantly towards the surface.
\end{abstract}

Keywords: Solitary wave, Chromatography, Trace element, Melt migration, Magma dynamics, Fluid migration

\footnotetext{
* Corresponding author

** Second orresponding author

Email addresses: jacob.jordan@yale.edu (Jacob S. Jordan), mhesse@jsg.utexas.edu (Marc A. Hesse)
} 


\section{Introduction}

Fluid migration in ductile rocks controls important geological processes such as melt segregation and fluid expulsion during regional metamorphism. Fluid production by partial melting and devolatilization leads to a percolating fluid network that allows for the segregation of fluid by porous flow at very low porosities (von Bargen and Waff, 1986; Cheadle, 1989; Wark and Watson, 1998 Miller et al. 2014, Ghanbarzadeh et al., 2014). Fluid segregation is driven by the buoyancy of the fluid and resisted by viscous compaction of the solid matrix (McKenzie, 1984, Scott and Stevenson, 1984, Fowler, 1985a). Fluid flow in rocks is predominantly vertical, because the segregation velocity of the fluid is significantly faster than the solid state creep velocity of the ductile rocks (Phipps Morgan, 1987, Sparks and Parmentier, 1991; Katz, 2008).

Fluid production in heterogeneous rocks leads to spatial variations in fluid content that may evolve into porosity waves, which migrate upwards at a velocity greater than the segregation velocity of the buoyantly rising background fluid. Porosity waves are an ubiquitous feature of the equations governing melt migration by porous flow (Spiegelman, 1993c). Porosity waves are also thought to „arise from fluid expulsion during regional metamorphism (Bailey, 1990; ThompSon and Connolly, 1990, Connolly, 1997, 2010, Tian and Ague, 2014, Skarbek and Rempel, 2016) and in the context of brine and hydrocarbon migration in sedimentary basins (McKenzie, 1987, Connolly and Podladchikov, 2000, Appold and Nunn, 2002, Joshi and Appold, 2016). In the aforementioned applications it is important to understand if solitary waves are effective carriers of energy, mass and geochemical signals. Here we revisit the viability of transport by porosity waves.

An idealized limit of compaction-driven porosity waves are so-called solitary porosity waves, which propagate at constant phase velocity, $\lambda$, without change in shape (Figure $1 a$ ). In solitary waves the decompaction due to fluid overpressure at the front is perfectly balanced by compaction due to fluid underpressure in the back (McKenzie, 1984, Scott and Stevenson, 1984, 1986, Barcilon and Richter 

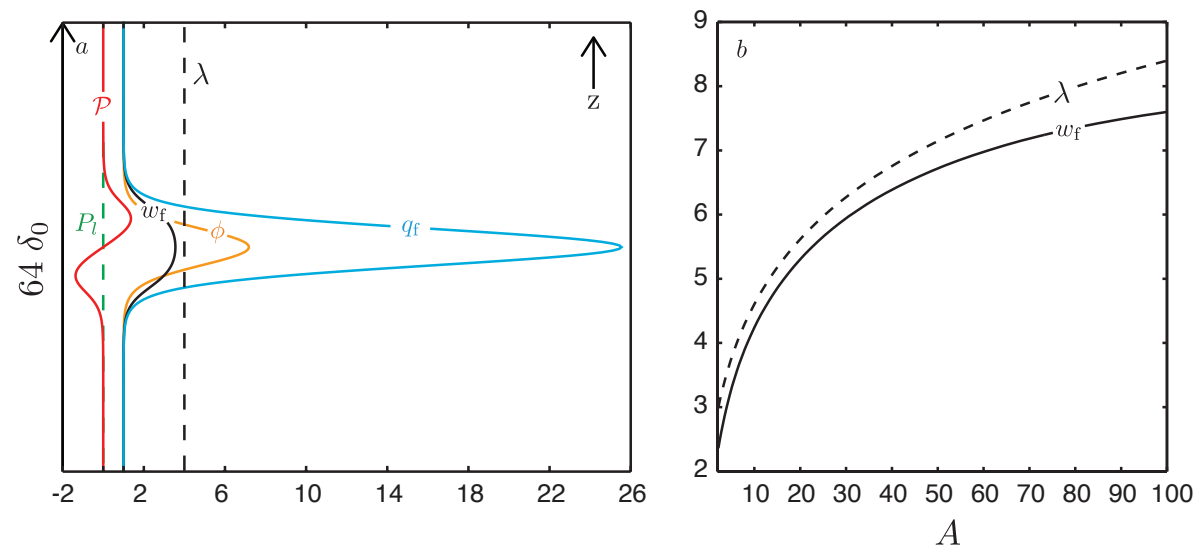

Figure 1: One dimensional solitary porosity wave with phase speed, $\lambda=4$. a) A high accuracy numerical solution for a dimensionless, one dimensional solitary porosity wave from Simpson and Spiegelman (2011): Porosity, $\phi$, is scaled to the background porosity, $\phi_{0}=0.001$. Fluid pressure, $\mathcal{P}$ is scaled by the pressure due to buoyancy over the characteristic length scale, $\Delta \rho g \delta_{0}$. In the ambient background $\mathcal{P}$ is the lithostatic pressure, $P_{l}$. The upward volumetric flux of the fluid, $q_{\mathrm{f}}$, and its vertical velocity $w_{\mathrm{f}}$ are scaled to the background separation flux, $q_{0}$. Both $q_{\mathrm{f}}$ and $w_{\mathrm{f}}=q_{\mathrm{f}} / \phi$ are elevated within the solitary porosity wave. b) Phase and vertical fluid velocities as functions of amplitude, $A$, of the porosity increase at the center of the solitary porosity wave. All calculations use the constitutive exponents $(n, m)=(2,1)$, see Section 2.1 for definition. 1986, Wiggins and Spiegelman, 1995, Simpson and Spiegelman, 2011). In one

36 Lovera, 1989, Watson and Spiegelman, 1994, Spiegelman, 1994, Liang, 2008,

37 Solano et al., 2014). This analysis of the one-dimensional case has led to the

38 assumption that porosity waves in general cannot transport mass.

In addition, fluid transport by porous flow in local chemical equilibrium 40 leads to chromatographic separation of chemical elements according to their ${ }_{41}$ compatibility within the solid matrix (McKenzie, 1984, Navon and Stolper, 1987.

42 Richter and Daly, 1989). A perfectly incompatible element travels at the velocity 


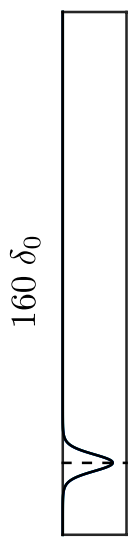

$$
t=0
$$
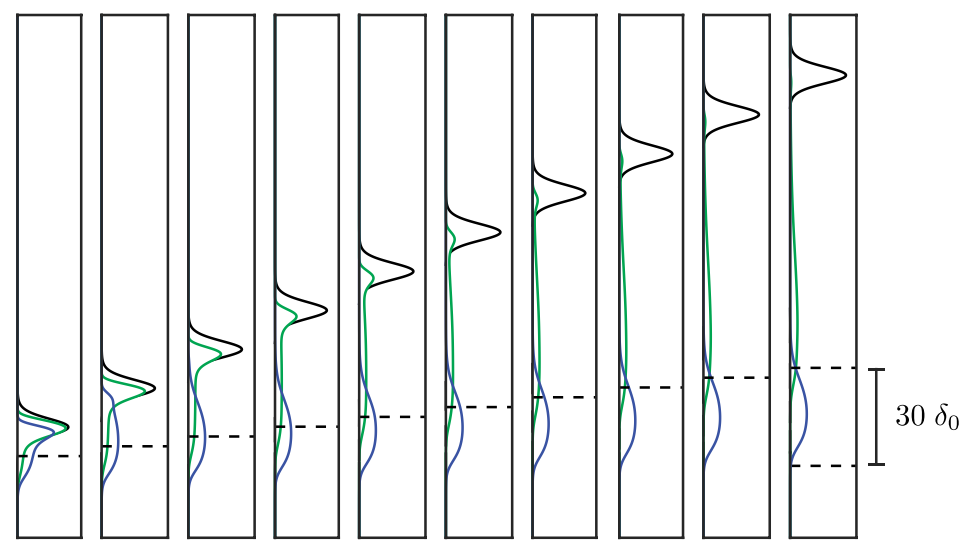

$\rightarrow t=30$

Figure 2: Tracer transport in a one dimensional solitary porosity wave, for animation see supp_2.avi. Two chemical tracers of different compatibility are initially co-located with the porosity anomaly due to melting of a heterogeneity. The green tracer is perfectly incompatible $(D=0)$ with the solid phase and travels at the velocity of the melt. The blue tracer is slightly compatible $\left(D=2 \times 10^{-3}\right)$ with the solid and travels with a reduced velocity. For definition of distribution coefficient, $D$, see Section 3 Time is scaled to the amount of time required for the background melt to travel one characteristic compaction length, $\delta_{0}$. Characteristic scales are introduced in Appendix Appendix A.3 The distance melt travels at the characteristic velocity is demonstrated by the dotted black line and four times slower than the solitary porosity wave traveling at the phase speed $\lambda=4$. All calculations use the constitutive exponents $(n, m)=(2,1)$, see Section 2.1 for definition.

Fluid transport with porosity waves and chromatographic separations appear 49 to make it impossible to preserve the distinct geochemical signature associated so with the source region of the fluid. This is illustrated by the numerical simula${ }_{51}$ tion shown in Figure 2. Here, fluid production has locally increased porosity and ${ }_{52}$ is initially co-located with two associated trace elements. Although the region of 
elevated porosity and trace element concentration are initially co-located, they become separated during fluid migration. As the trace element signatures abandoned by the porosity wave slowly migrate upwards, the continuous exchange between the fluid and solid separates tracers according to their compatibility. This implies that transport induced by the increase in fluid supply due to local fluid production carries with it no distinct geochemical signature.

However, the conclusion that solitary porosity waves do not transport mass is based upon one dimensional studies of melt transport. It is well known that onedimensional porosity waves are unstable in two and three dimensions and break up into sets of cylindrical or spherical porosity waves Scott and Stevenson, 1986, Wiggins and Spiegelman, 1995). Here we show that tracer transport in such higher dimensional porosity waves is dramatically different that in one dimension.

\section{Fluid flow in two dimensional porosity waves}

Models for fluid flow in ductile rocks assume a two phase mixture comprised of incompressible solid and melt phases. The flow of the fluid is described by Darcy's law and the solid matrix undergoes viscous deformation, often assumed to be Newtonian (McKenzie, 1984; Scott and Stevenson, 1984; Fowler, 1985a). Due to the intrinsic weakness of ductile rocks, porosities are very small. This allows significant simplifications to the governing equations that describe the two phase mixture. These simplified equations admit solutions in the form of solitary waves as shown in Figures 1 and 2 . The substantial literature on solitary wave solutions provides the ideal framework for discussing mass transport in porosity waves. 


\subsection{Governing equations in the small porosity limit}

The dimensionless governing equations for the evolution of a porosity anomaly in a uniform background, in the limit of small porosities, are

$$
\begin{aligned}
\frac{\partial \phi}{\partial t} & =\frac{\mathcal{P}}{\xi_{\phi}} \\
-\nabla \cdot K_{\phi} \nabla \mathcal{P}+\frac{\mathcal{P}}{\xi_{\phi}} & =-\nabla \cdot K_{\phi} \hat{\mathbf{z}}
\end{aligned}
$$

where $\mathcal{P}$ and $\phi$ are the dimensionless fluid pressure and porosity respectively and $\hat{\mathbf{z}}$ is the upward pointing unit vector. Here we write $1 \mathrm{a}$ in terms of the partial derivative rather than the material derivative and assume no net translation of the solid. For the full dimensional governing equations see Appendix A.1.

The dimensionless permeability, $K_{\phi}$, and effective viscosity, $\xi_{\phi}$, are functions of porosity based on phenomenological laws,

$$
K_{\phi}=\phi^{n} \quad \text { and } \quad \xi_{\phi}=\phi^{-m}
$$

where the values of the exponents are typically $n \in(2,3)$ and $m \in(0,1)$, Wark and Watson, 1998, Simpson and Spiegelman, 2011).

The porosity has been scaled to the characteristic porosity, $\phi_{0}$, of the ambient background outside the porosity anomaly. The natural length scale that arises from the governing equations is the compaction length of the background, $\delta_{0}=\sqrt{K_{0} \xi_{0} / \mu}$, where $K_{0}$ and $\xi_{0}$ are permeability and effective viscosity of the background and $\mu$ is the fluid viscosity.

The fluid pressure, $\mathcal{P}$, is scaled by the pressure due to buoyancy over a compaction length, $\Delta \rho g \delta_{0}$, where $\Delta \rho=\rho_{\mathrm{s}}-\rho_{\mathrm{f}}$ is the density difference between solid and fluid, and $g$ is the gravitational acceleration. The sign of $\mathcal{P}$ therefore indicates over and underpressure. Time is scaled by the segregation time $\delta_{0} / w_{0}$, where the segregation velocity $w_{0}=K_{\phi} \Delta \rho g / \phi_{0} \mu$, is induced by the buoyancy of the fluid. The characteristic time scale is the time required for a percolating fluid to traverse a compaction length in the background.

The governing equations (1) admit solitary wave solutions in one, two and three dimensions. Figure $3 a$ shows porosity contours and the fluid pressure for 
a two-dimensional solitary porosity wave. Due to buoyancy, the fluid in the upper half of the solitary porosity wave is above lithostatic pressure and dilates the matrix, while the pressure of the fluid in the lower half is below lithostatic, allowing the matrix to compact. This balance between dilation and compaction leads to steady upward migration of the solitary porosity wave at a fixed phase speed, with solutions when $\lambda \geq 3$. Figure $3 a$ also shows a cross-section of the two dimensional plot to help draw comparison to Figure 1. Below we utilize two dimensional solutions for solitary porosity waves with $(n, m)=(2,1)$ provided by Simpson and Spiegelman (2011), to highlight previously unrecognized implications for mass transport in porosity waves.

\subsection{Mass transport mechanism in solitary porosity waves}

To understand mass transport within solitary porosity waves, the fluid and solid flow fields must be computed. Although the governing equations in the small porosity limit are independent of the solid flow field, knowledge of the solid flow field is required to understand the transport of compatible trace elements. The movement of the solid can be recovered by solving the following equation for the scalar solid velocity potential,

$$
-\nabla^{2} \mathcal{U}=\frac{\mathcal{P}}{\xi_{\phi}} .
$$

The potential $\mathcal{U}$ captures the perturbation to the solid velocity field from compaction and decompaction induced by the solitary porosity wave. Once $\mathcal{U}$ and $\mathcal{P}$ are known, the flux of fluid relative to solid, $\mathbf{q}_{\mathrm{r}}$, is described by Darcy's law and the solid velocity field, $\mathbf{v}_{s}$, is found from the gradient of the solid velocity potential,

$$
\mathbf{q}_{\mathrm{r}}=\phi \mathbf{v}_{\mathrm{f}}=-K_{\phi}[\nabla \mathcal{P}-\hat{\mathbf{z}}] \quad \text { and } \quad \mathbf{v}_{\mathrm{s}}=-\nabla \mathcal{U}
$$

In the small porosity limit, the motion of the solid can be neglected in the formulation of Darcy's law, so that the fluid flux is equal to the relative fluid flux, $\mathbf{q}_{\mathrm{f}}=\mathbf{q}_{\mathrm{r}}$ (see Appendix A.3. Throughout this study, $u$ represents the horizontal component of the velocity field and $w$ denotes the vertical $\mathbf{v}_{\mathrm{p}}=\left[u_{\mathrm{p}} w_{\mathrm{p}}\right]$, where 

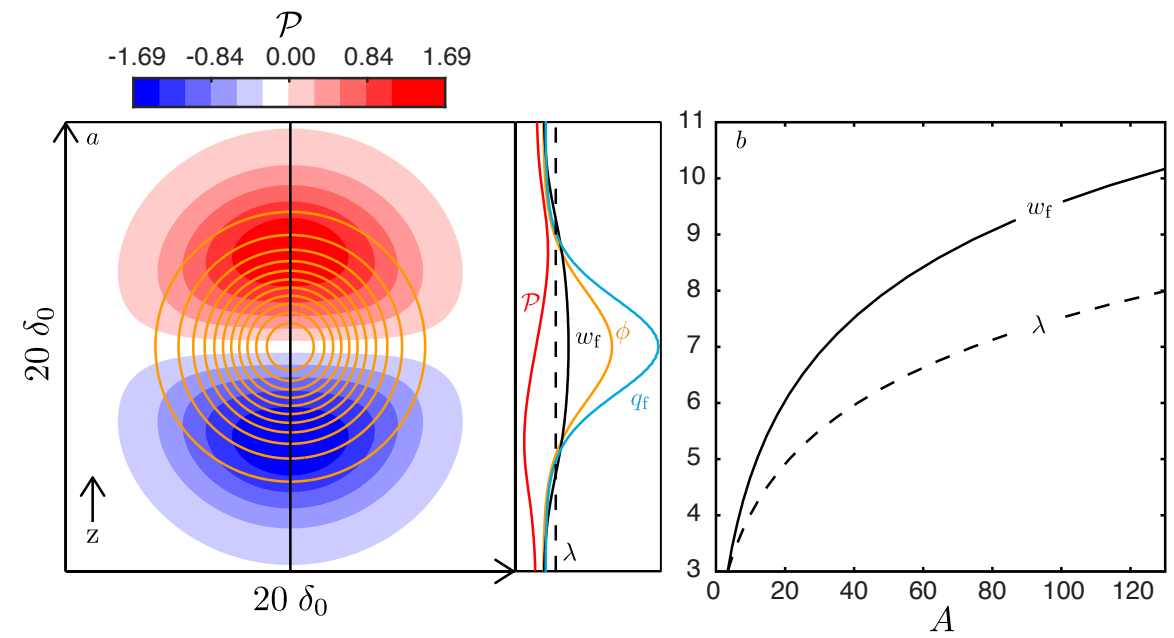

Figure 3: a) Two-dimensional solitary porosity wave with phase speed, $\lambda=4$, and constitutive exponents, $(n, m)=(2,1)$. Dimensionless pressure of the melt phase, $\mathcal{P}$, with porosity contours, $\phi$ in gold from Simpson and Spiegelman (2011). The black line down the center of the contour plot shows the spatial location of the "one-dimensional" profile. This profile is analogous to Figure $11 a$. Notably, $w_{\mathrm{f}}>\lambda$ suggesting that sustained mass transport is possible within two-dimensional solitary porosity waves. b) Vertical melt velocity at the center of the solitary porosity wave and phase speed as a function of amplitude, $A$. Constitutive exponents, $(n, m)=(2,1)$ as in $a$.

subscript, $\mathrm{p} \in[\mathrm{f}, \mathrm{s}]$ denotes the fluid and solid phases. Unlike previous studies, the solid velocity has been scaled by $\phi_{0} w_{0}$. This scaling takes into account the reduction of solid motion with declining background porosity.

The fluid flux, $\mathbf{q}_{\mathrm{f}}$, in both one and two dimensional solitary porosity waves is enhanced relative to the background, $q_{0}=\phi_{0} w_{0}$. Similar to the one dimensional case, the phase velocity of the porosity wave is larger than the background fluid velocity (Figures $1 a$ and $3 a$ ). In one dimension, fluid speed never exceeds the phase speed of the porosity wave. Therefore, an incompatible tracer experiences no sustained transport (Figure 1 and Figure 22). In contrast, Figure $3 b$, shows that the fluid velocity at the center of two dimensional velocity waves exceeds the phase velocity, $w_{\mathrm{f}}>\lambda$ for all $\lambda>3$.

Figures $4 a$ and $4 b$ show the streamlines of the solid and fluid velocity fields, 
$\mathbf{v}_{\mathrm{S}}$ and $\mathbf{v}_{\mathrm{f}}$ in a fixed Eulerian reference frame at an instant in time. In the Eulerian reference frame the solid in the far-field is stationary. Solid streamlines show a dipole-like pattern. They emanate from the dilating region at the front of the porosity wave, and converge in the compacting region at the back. Fluid streamlines are sub-vertical and deflected towards the interior of the solitary porosity wave, indicating a focusing of the fluid flux into the high porosity wave. Focusing of the flow allows the speed of the fluid at center of the wave to exceed the phase speed (Figure $3 b$ ). This suggests that sustained tracer transport may be possible, because perfectly incompatible tracers at the center of the wave move faster than the solitary porosity wave and are not left behind as in Figure 2 .

However, in the Eulerian reference frame it is not possible to infer the physical path of fluid from the streamlines, because the porosity field and its associated velocity fields evolve in time. In a Lagrangian reference frame, moving with the constant phase speed of the solitary porosity wave,

$$
\tilde{w}_{\mathrm{p}}=w_{\mathrm{p}}-\lambda, \quad \mathrm{p} \in[\mathrm{f}, \mathrm{s}]
$$

the porosity field and the streamlines become stationary (Figure $4 c$ and $4 d$ ). Here, the solid streamlines are sub-vertical and deflected outward from the center of the porosity wave. In the far-field, the solid moves downward with speed $\lambda$.

After shifting into the Lagrangian reference frame, the movement of the fluid becomes apparent. Fluid streamlines show distinct behavior in the interior and exterior of the porosity wave (Figure $4 d$ ). These regions are separated by two semi-circular dividing streamlines that meet at two stagnation points along the vertical symmetry axis of the porosity wave, where $\left(u_{\mathrm{f}}, \tilde{w}_{\mathrm{f}}\right)=0$. In the interior, there are two symmetric cells of closed streamlines where fluid circulates outwards around two additional stagnation points along the horizontal axis of symmetry. Outside the circular dividing streamline, the fluid streamlines are sub-vertical and deflected away from the wave moving downward at a speed bounded between 0 and $\lambda$, relative to the background velocity of the solid. Fluid 

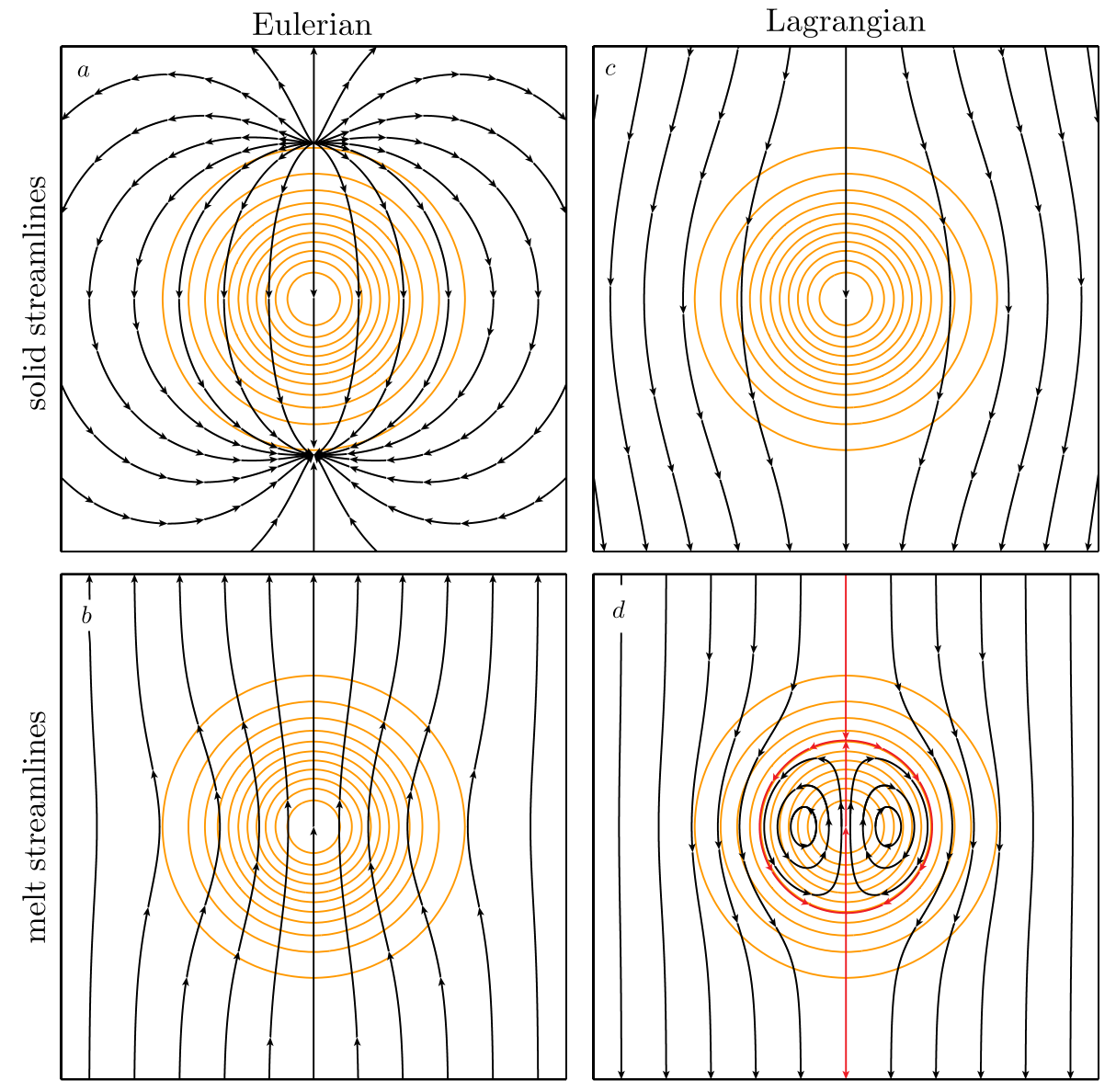

Figure 4: Streamlines of a solitary porosity wave with $\lambda=4$ and constitutive exponents $(n, m)=(2,1)$. The porosity contours in gold are identical to those shown in Figure 3a. a) Solid flow streamlines in Eulerian reference frame. b) Fluid streamlines in Eulerian reference frame. c) Solid streamlines in Lagrangian reference frame. d) Fluid streamlines in Lagrangian reference frame, $\tilde{w}_{\mathrm{p}}=w_{\mathrm{p}}-\lambda$, where $\mathrm{p}=(\mathrm{f}, \mathrm{s})$. Dividing streamlines are depicted in red. Within the dividing streamline there are symmetrical counter-rotating cells. Each panel is $20 \delta_{0} \times 20 \delta_{0}$ 
on the interior of the circular dividing streamline is transported by the solitary porosity wave, while fluid outside is stripped away and experiences no sustained transport. Unlike their one dimensional counterparts, two dimensional porosity waves may transfer mass.

\section{Tracer transport in porosity waves}

Starting with Korzhinskii (1965) and Hofmann (1972) it has been recognized that elements may undergo chromatographic separation during fluid percolation in geological processes. In magmatic systems, chromatographic separations are most commonly invoked in studies of trace element and radionuclide transport (McKenzie, 1984, Navon and Stolper, 1987, McKenzie, 1985a, De Paolo, 1996 , Hauri, 1997; Hauri and Kurz, 1997). Similarly, chromatographic concepts have been important in metamorphic systems, where they are commonly applied to stable isotope transport (Norton and Taylor, 1979, Baumgartner and Rumble III, 1988, Bickle and McKenzie, 1987, Bowman and Willett, 1991). In either case, models with one-dimensional flow at constant porosity result in the linear separation of tracers based on their compatibility with the solid phase.

\subsection{Tracer transport in low porosity limit}

The chromatographic separation of tracers is determined by the distribution coefficient, which is variably defined, either as a ratio of mass fractions (McSween et al. 2003), $D_{x}=x_{\mathrm{s}} / x_{\mathrm{f}}$, or as a ratio of concentrations (White, 2013),

$$
D=\frac{c_{\mathrm{s}}}{c_{\mathrm{f}}}=\frac{\rho_{\mathrm{s}} x_{\mathrm{s}}}{\rho_{\mathrm{f}} x_{\mathrm{f}}}=\frac{\rho_{\mathrm{s}}}{\rho_{\mathrm{f}}} D_{x}
$$

Here, $c_{\mathrm{S}}$ is the total concentration of the tracer in all solid phases and $c_{\mathrm{f}}$ is its concentration in the fluid and similarly $x_{\mathrm{s}}$ is the total mass fraction of tracer partitioned into all solid phases while $x_{\mathrm{f}}$ is the mass fraction of tracer in the fluid. For a perfectly incompatible tracer $D=0$, there is no incorporation of the trace element into the solid phase and the velocity of the tracer is that of 
the fluid flow field (Figures $4 \beta$ and $4 d$ ). Conversely, as $D \rightarrow \infty$, the tracer prefers the solid and the effective velocity of the tracer is that of the solid flow field (Figures $4 a$ and $4 c$ ). For all intermediate cases, the dimensionless effective velocity in the small porosity limit is given by

$$
\mathbf{v}_{\mathrm{e}}=\frac{\phi \mathbf{v}_{\mathrm{f}}+\mathbf{v}_{\mathrm{s}} D}{\phi+D / \phi_{0}},
$$

where a term containing the characteristic porosity, $D / \phi_{0}$, has been retained, because the distribution coefficient itself may be small. Here, the dimensionless effective tracer velocity is scaled by $w_{0}$. For dimensional equations, scaling and simplification see Appendix A.2, Appendix A.3 and Appendix A.4 respectively. When $D \ll \phi_{0} \ll 1, \mathbf{v}_{\mathrm{e}} \rightarrow \mathbf{v}_{\mathrm{f}}$ and when $D \gg \phi_{0}, \mathbf{v}_{\mathrm{e}} \rightarrow \phi_{0} \mathbf{v}_{\mathrm{s}} \approx 0$. Figure 2 illustrates the reduction in the effective velocity of a moderately compatible tracer relative to a perfectly incompatible tracer in a one dimensional flow field.

Assuming chemical equilibrium and purely advective transport, the dimensionless conservation equation for bulk tracer evolution in absence of hydrodynamic dispersion is given by

$$
\frac{\partial \mathcal{C}}{\partial t}+\nabla \cdot\left[\mathbf{v}_{\mathrm{e}} \mathcal{C}\right]=0,
$$

where the dimensionless bulk concentration of tracer in the small porosity limit is given by

$$
\mathcal{C}=\left(\phi+D / \phi_{0}\right) x_{\mathrm{f}} .
$$

${ }_{174}$ For the derivation and scaling of equations (8) and (9) see appendices Appendix A.2 to Appendix A.4. Below, we first investigate the evolution of a perfectly incompatible tracer in the fluid phase, before illustrating the effect of partitioning on tracer transport by porosity waves.

\subsection{Perfectly incompatible tracer}

Consider a local increase in porosity generated by localized melting or fluid production characterized by a distinctive geochemical tracer, $\mathcal{C}$, as shown in the $t=0$ panel of Figure 5 According to conventional wisdom, the tracer should 


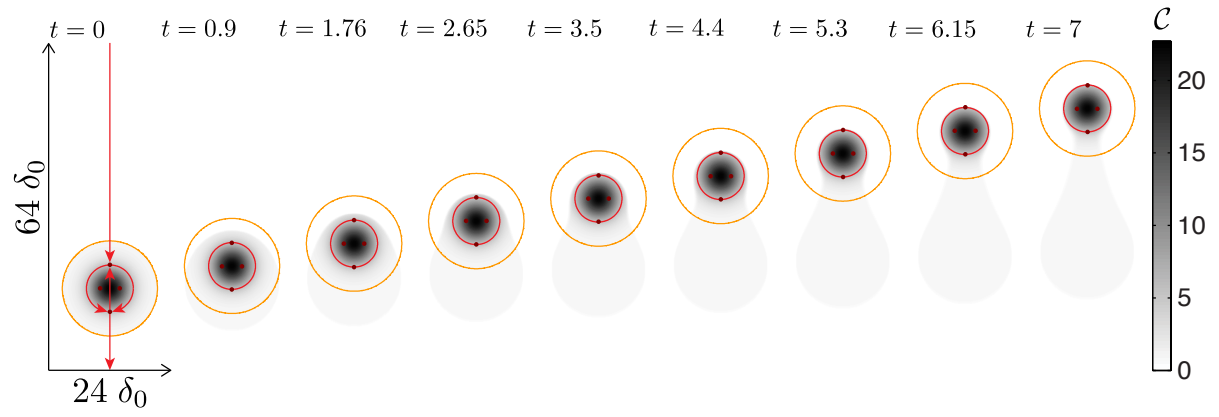

Figure 5: Demonstration of transport of bulk composition, $\mathcal{C}$, for a perfectly incompatible tracer, $D=0$, initially co-located with a solitary porosity wave $(\lambda=5,(n, m)=(2,1))$. This simulation uses the same governing equations and constitutive relationships as in Figure 2 Red lines with arrowed tips show the dividing streamlines and their direction of flow. Maroon dots show the location of stagnation points where $u_{\mathrm{f}}=\tilde{w}_{\mathrm{f}}=0$. Lastly, the gold circle is five percent of the maximum porosity anomaly. Within this gold circle the tracer field is initialized to $x_{\mathrm{f}}=1$. For an incompatible tracer the dimensionless bulk composition is simply, $\mathcal{C}=\phi x_{\mathrm{f}}$, so the variation of $\mathcal{C}$ within the wave is largely a reflection of dimensionless porosity field, $\phi$.

become decoupled from the porosity wave, similar to the one-dimensional case shown in Figure 2. However, the results in Figure 5 illustrate that only the outer portion of the tracer is stripped away, while the tracer in the center migrates upward with the solitary porosity wave. This central region corresponds to the area within the circular dividing streamline. Once the porosity wave migrates a distance proportional to the radius of the circular dividing streamline, it will contain two distinct fluids: one derived from the heterogeneity that generated the solitary porosity wave and another from the background.

To more effectively illustrate the motion of the tracer that is transported by the solitary porosity wave, consider the initial tracer distribution shown in the first panel of Figure 6. Here, the initial distribution of the tracer is confined to a disc that is smaller than the circular dividing streamline. In this case, the porosity wave transports two fluids of distinct composition within the circular dividing streamline, distinguished by the concentration of the tracer, $\mathcal{C}$. The circulation of the fluid within the dividing streamline is much faster than the phase speed of the solitary porosity wave. This stretches the tracer 


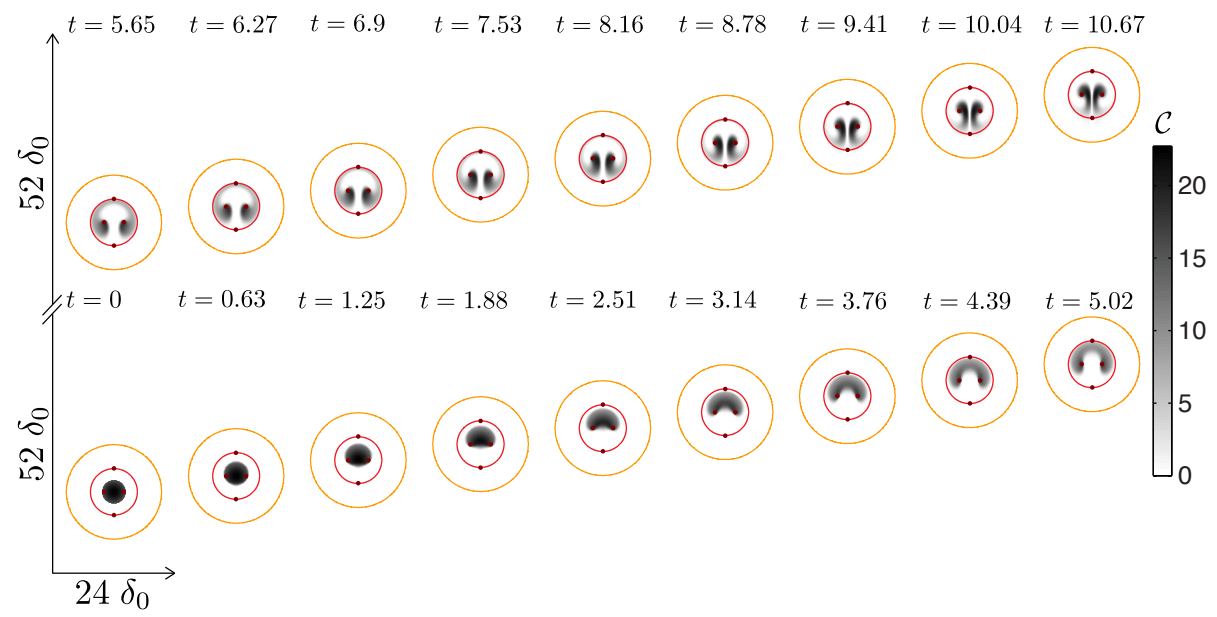

Figure 6: Transport of bulk composition, $\mathcal{C}$, using initial tracer distribution confined to disc smaller than the circular dividing streamline of a solitary porosity wave $(\lambda=5$, $(n, m)=(2,1))$. The initial condition is, $x_{\mathrm{f}}=1$ for $\phi>0.7 \max (\phi)$ and zero elsewhere. Circular dividing streamline is shown in red, stagnation points are in maroon and the gold halo representing the five percent porosity contour is left for comparison to Figure 5

into symmetrical filaments that spiral inwards to the central stagnation points in a swirling motion reminiscent of Cinnamon rolls, similar to tracer patterns pbserved solitary waves that arise in in Stokes flows in fluid conduits (Whitehead and Helfrich, 1988).

The tracer pattern in Figure 6 remains relatively sharp, because molecular diffusion, $\mathrm{D}_{\mathrm{mol}}$ and mechanical dispersion, $\mathrm{D}_{\mathrm{dis}}$, are neglected in the simulation. The presence of any significant hydrodynamic dispersion $D_{\text {hyd }}=D_{\text {mol }}+D_{\text {dis }}$, will tend to homogenize the composition of the fluid transported within the porosity wave. The importance of hydrodynamic dispersion is given by the Péclet number, $\mathrm{Pe}=w_{\mathrm{m}} r / \mathrm{D}_{\text {hyd }}$, where a suitable length scale is the radius, $r$, of the circular dividing streamline. Dispersion could result in the homogenization of the tracer within smaller porosity waves during migration. Furthermore, small amounts of tracer may be lost to the background across the dividing streamline when hydrodynamic dispersion is considered. The importance of dispersion is difficult to asses, because the physical size of solitary porosity 
waves changes dramatically with the choice of the constitutive exponents, $n$ and $m$. For discussion of this issue see Section 4.2

\subsection{Tracers of varying compatibility}

Tracers with nonzero distribution coefficients are transported by an effective velocity field that is a weighted average of the solid and fluid flow fields, given by equation (7). The Lagrangian fluid streamlines for tracers with increasing distribution coefficients are shown in Figure 7. The overall circulation pattern remains the same, except the radius of the circular dividing streamline shrinks with increasing $D$ as the effective velocity decreases. At the critical distribution coefficient, $D^{*}$, the circular dividing streamline has collapsed to a point and the solitary porosity wave stops transporting the tracer.

This implies that the effect of partitioning on tracer transport in higher dimensional solitary porosity waves is drastically different from transport in one-dimensional columns typically considered. In one dimension the distribution coefficient determines velocity of transport. In contrast, within higher dimensional solitary porosity waves the distribution coefficient determines the amount of tracer transported, but not its overall velocity. Of course, the migration of compatible trace elements along the circular streamlines is retarded, but the overall vertical migration velocity is $\lambda$ for all trace elements with $D<D^{*}$. Trace elements with $D \geq D^{*}$, are not transported by solitary porosity waves.

The exact value of $D^{*}$ depends on the phase speed and amplitude, of the solitary porosity wave, as shown in Figure $8 a$ and on the constitutive exponents, $n$ and $m$. As the distribution coefficient increases above $D / \phi_{0}=10^{-1}$ the volume of fluid transporting tracer, $V_{\mathrm{e}}$, begins to decrease and vanishes at $D^{*}$. For transport to occur the vertical effective velocity of the tracer, $w_{\mathrm{e}}$, must exceed the phase speed, $\lambda$, of the solitary porosity wave. Since the fluid velocity is largest in the center of the wave, $D^{*}$ can be obtained from (7) by setting $w_{\mathrm{e}}=\lambda$ at the center of the solitary porosity wave. Therefore, the critical 


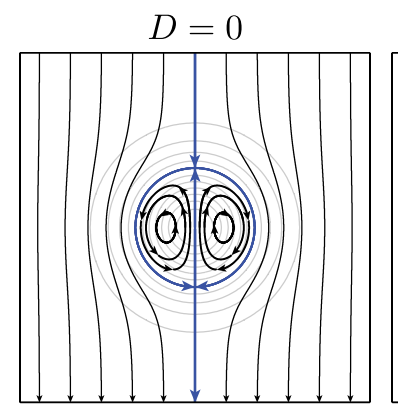

$D / \phi_{0}=1$
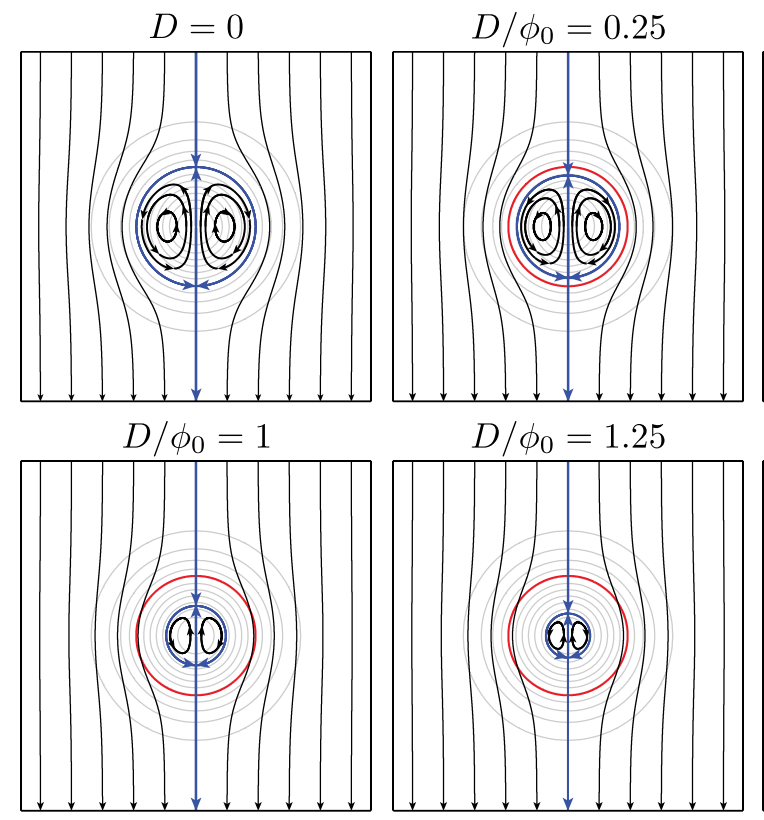

$D / \phi_{0}=1.25$
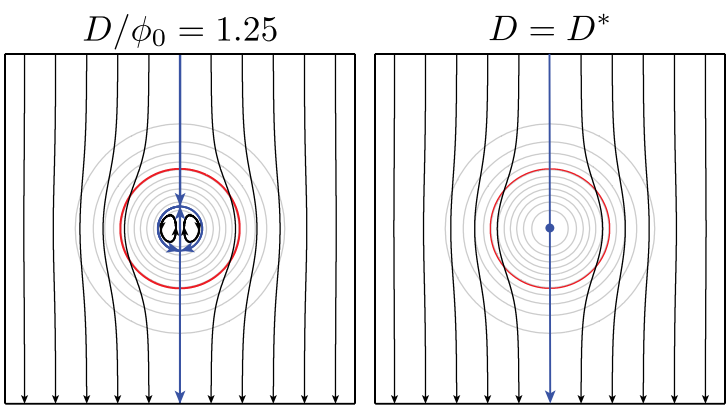

Figure 7: Demonstration of increasing distribution coefficient $D$ on fluid streamlines within a solitary porosity wave $(\lambda=5,(n, m)=(2,1))$. Fluid streamlines are shown in black with the dividing streamline in blue. The red circle indicates the dividing streamline for the perfectly incompatible incompatible case, $D=0$, for reference. As $D$ increases the semicircular dividing streamline of the effective velocity shrinks until it vanishes at the critical distribution coefficient $D^{*}$. At $D^{*}$ the vertical fluid velocity of the wave in the Lagrangian reference frame $\tilde{w}_{\mathrm{e}}=0$. The blue dot in the $D^{*}$ panel indicates the point that the vertical velocity profile becomes zero a the porosity maximum for a tracer with compatibility $D^{*}$. As in Figures 3 and the wave speed, $\lambda=4$. The size of the domain in all cases is $20 \delta_{0} \times 20 \delta_{0}$. 

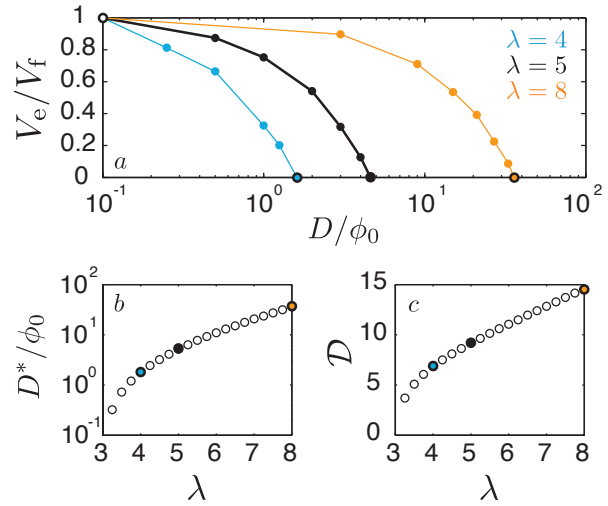

Figure 8: a) Normalized volume of tracer melt retained by two dimensional solitary porosity waves as a function of partitioning behavior and phase speed. Light blue circles for $\lambda=4$, correspond to results shown in Figure 7 The intercept at the x-axis where $V_{\mathrm{e}}=0$ corresponds with $D^{*} . b$ ) The phase speed of the critical partition coefficient, $D^{*}$ against phase speed, $\lambda$. Filled in, colored, circles correspond to the x-intercept of 8 a. For visualization of the growth of $\mathcal{D}$ with $\lambda$ and the corresponding porosity profile, see Figure B.12 in Appendix B

distribution coefficient is given by

$$
D^{*} / \phi_{0}=\phi\left(w_{\mathrm{f}} / \lambda-1\right) \quad \text { at } \quad \tilde{\mathbf{x}}=\mathbf{0},
$$

where the values of the variables at the center are obtained from the semianalytical solution for the solitary porosity wave (Simpson and Spiegelman 2011). Figure $8 b$ shows that $D^{*}$ increases rapidly with $\lambda$, because larger amplitude porosity wave focus fluid more effectively. For distribution coefficients based on mass fractions the critical distribution coefficient is $D_{x}^{*} \sim \rho_{\mathrm{f}} / \rho_{\mathrm{s}} D^{*}$.

Due to lithological changes, partitioning behavior often changes with depth as a porosity wave rises buoyantly. While the porosity wave itself is not affected by partitioning, the radius of the dividing streamline changes. Figure 9 illustrates the resultant mixing behavior assuming a sharp decrease in $D$. Below the transition, the dynamics of tracer transport are analogous to the behavior shown in Figure 5. However, due to the nonzero distribution coefficient, the radius of the circular dividing streamline is smaller (Figure 7), resulting in a reduced volume within which tracer is transported (Figure 8a). As the solitary porosity 


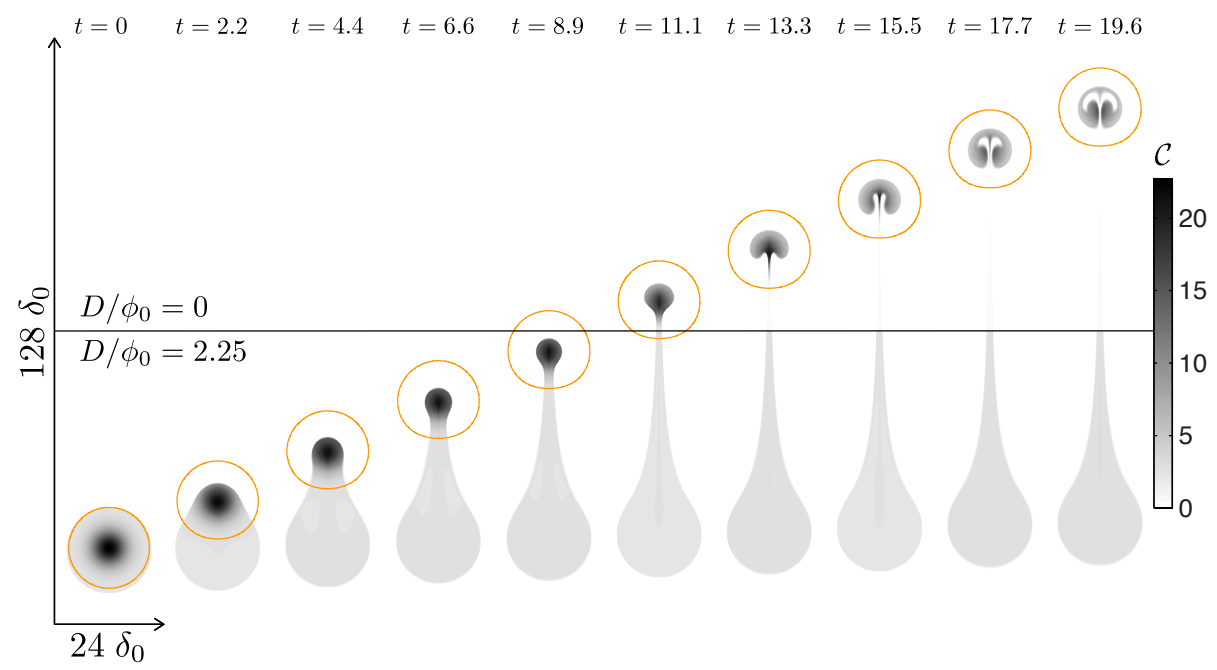

Figure 9: Finite volume simulation initialized with solitary wave solution used in Figures 5 and 6 For animation see supp_9.avi. The gray-scale filled contours show the evolution of tracer composition. As in Figure 5 the gold circle is five percent of the maximum porosity anomaly. Half way through the domain, at sixty four characteristic compaction lengths, the compatibility of the tracer with the solid matrix transitions from $D / \phi_{0}=2.25$ to perfectly incompatible, $D=0$, as indicated at the left hand side of the figure.

wave crosses the transition in the distribution coefficient, the expansion of the dividing streamline incorporates background fluid into the transported volume. This newly incorporated fluid, is primarily derived from above the transition and becomes mixed with the tracer carried from below in a swirling pattern analogous to the dynamics in Figure 6. The migration of solitary porosity waves across such transitions in mineral assemblage therefore provides a natural mixing mechanism for fluids with different trace element signatures and different depths of origin.

\section{Discussion}

For clarity, the analysis presented in this manuscript is based upon highly idealized solutions for solitary porosity waves. These waves have been studied extensively and their properties are well constrained (Scott and Stevenson, 
1984, 1986, Barcilon and Richter, 1986, Barcilon and Lovera, 1989; Richter and McKenzie, 1984, Richter and Daly, 1989, Wiggins and Spiegelman, 1995, Simpson and Spiegelman, 2011). For the Lagrangian reference frame used in this study, knowledge of the exact phase velocity of the porosity waves is essential. The results above show that solitary porosity waves in two dimensions transport mass. Below we use numerical simulations to demonstrate that this conclusion also applies to a broader set of less idealized porosity waves and their formation. This is followed by a comparison of tracer transport in two-dimensional porosity waves and classical results in one-dimensional linear columns. Finally, we discuss the physical dimensions and transport timescales of the porosity waves in ductile rocks as well as the limitations of this model.

\subsubsection{Tracer incorporation during formation of porosity waves}

The examples discussed in Section 3.2 demonstrate that an incompatible tracer can be transported by a fully formed, two-dimensional solitary porosity wave. In all examples shown above the tracer is initially located in the recirculating region within the circular dividing streamline. In these simulations fluid at the center of the solitary porosity wave is isolated from the background for the entire duration of transport. Therefore, it is not yet clear if a tracer can be incorporated into a porosity wave during its formation.

To illustrate the incorporation of a tracer into porosity waves, we study the break-up of a perturbed one-dimensional solution representing a laterally extensive region of elevated porosity. Several authors have shown that onedimensional solitary porosity waves are unstable in higher dimensions and lead to the formation of stable, higher-dimensional porosity waves (Scott and Stevenson, 1986, Wiggins and Spiegelman, 1995). Figure 10 shows the evolution of a perturbed one-dimensional solitary porosity wave from Simpson and Spiegelman (2011) in two-dimensions. The unperturbed one-dimensional evolution of this initial condition using the same parameters is shown in Figure 2, which demonstrates that tracers are not transported. If the two-dimensional simulation is not perturbed, the solution remains one-dimensional and reproduces the behav- 


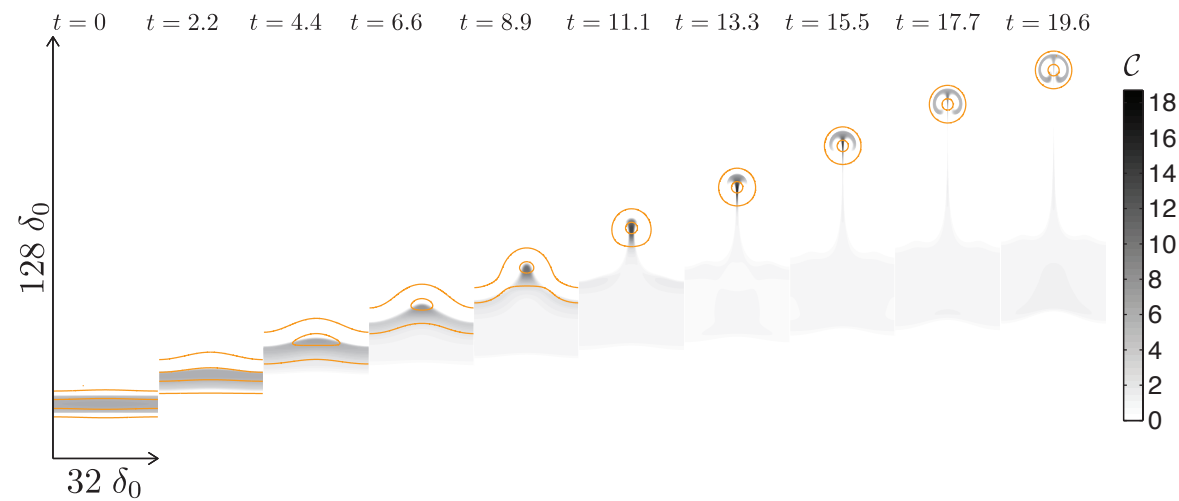

Figure 10: Finite volume simulation initialized with a transversely perturbed one-dimensional solution for a solitary porosity wave, for animation see supp_10.avi. The initial condition corresponds to the one-dimensional solitary porosity wave $(\lambda=4,(n, m)=(2,1))$ from Simpson and Spiegelman (2011), also shown in Figure 2 Tracer is initialized so that $x_{\mathrm{f}}=1$ where $\phi$ is greater than $50 \%$ of the maximum porosity anomaly. Contours for $20 \%$ and $80 \%$ of the maximum initial porosity anomaly are shown in gold.

ior seen in Figure 2. However, a slight perturbation in porosity leads to the break-up of this one-dimensional wave and the formation of a two-dimensional porosity wave.

Figure 10 shows that some tracer is initially left behind, while the wave remains quasi-one-dimensional. Eventually, the wave-front scallops and forms a porosity maximum in the center of the domain due to the perturbation. This central porosity maximum begins to collect fluid laterally, increasing the fluid velocity and propelling the tracer upwards. The local increase in vertical fluid velocity leads to the incorporation of a fraction of the tracer originally co-located with the one-dimensional solitary porosity wave. Meanwhile, the porosity field coalesces into a radially symmetric porosity wave that travels with constant velocity and asymptotes towards solitary wave behavior as described in Section 2 Within this porosity wave a swirling tracer mixing pattern develops similar to Figure 6. This suggests that the formation of porosity waves leads to the incorporation and mixing of geochemical signatures initially located within the one-dimensional porosity wave with that of the ambient background near the 
initial location.

The porosity wave forming in Figure 10 is not a true solitary wave in the mathematical sense, but the dynamics of tracer transport are similar to the limiting case of a true solitary porosity wave. This demonstrates that a broader set of porosity waves recirculate fluid in the interior and therefore allow mass and tracer transport. Given that wave-like behavior is ubiquitous in simulations of fluid flow in ductile ductile rocks, this transport mechanism applies to a broad range of geological phenomena, including: partial melting and melt segregation in the mantle (Katz and Rudge, 2011, Weatherley and Katz, 2012) fluid release during regional metamorphism (Bailey, 1990, Thompson and Connolly, 1990 Connolly, 1997, 2010, Tian and Ague, 2014, Connolly and Podladchikov, 2015 Skarbek and Rempel, 2016) and brine migration during compaction of sedimentary basins (McKenzie, 1987; Connolly and Podladchikov, 2000; Appold and Nunn, 2002, Joshi and Appold, 2016). These waves arise in a range of porous media as they are a consequence of the dispersive nature of the governing equations for fluid flow in a viscously compacting medium (Spiegelman, 1993ab).

\subsection{Implications for trace element transport in ductile rocks}

This manuscript shows that two-dimensional solitary porosity waves may transport mass and that trace element transport is possible when $D<D^{*}$. Increasingly compatible elements may be transported as phase speed and amplitude increase (Figure $8 b$ ). Tracer transport in porosity waves differs from one-dimensional tracer chromatography in several important ways. Classical chromatography in a linear flow field has the following characteristics:

1. Each element travels at a different velocity, determined by its distribution coefficient.

2. The absolute abundance of elements is not affected by chromatographic separation.

3. Linear chromatography provides no natural mechanism for mixing of distinct fluids. 
In contrast, transport in two dimensional porosity waves has the following characteristics:

1. Elements with $D<D^{*}$ are transported together with the velocity of the porosity wave, $\lambda w_{0}$.

2. The absolute abundance of elements transported with the wave is determined by compatibility.

3. Transport in porosity waves provides a natural mechanism for mixing of distinct fluids from different depths in a viscously compacting medium.

These differences arise because the transported fluid migrates along closed streamlines inside the porosity wave (Figure $4 d$ ). Along these closed streamlines chromatographic separation affects the angular velocity of tracers interacting with the solid phase, which only leads to a phase shift. This negates the chromatographic separations for sufficiently incompatible elements that are otherwise inevitable during fluid percolation. Instead of reducing the effective transport speed, increasing compatibility of a tracer reduces the diameter of the circular dividing streamline (Figure 7), thereby reducing the mass of tracer transported (Figure 8a). Therefore, mass transport in porosity waves may alter the relative abundances of trace elements with different compatibilities. The dynamics observed in Figures 9 and 10 demonstrate that fluid transport in porosity waves provides natural mechanisms to mix fluid of different origin and depth.

\subsection{Physical size and speed of porosity waves}

The size, $\Delta$, and velocity, $\Lambda$, of a porosity wave determine if the phenomena discussed here are relevant to a particular geologic process. Figure 11 a shows combinations of dimensional size and velocity for which porosity waves are expected, given typical upper-mantle parameters. Parameter values and calculations for Figure 11 are detailed in Appendix B.

The existence of porosity waves is limited to a diagonal band in logarithmic $\Delta \Lambda$-space, by the conditions that $\lambda>3$ and that the porosity is small. Here we assume that the small porosity approximation is valid to $5 \%$ porosity, so that 
$\phi_{\max }=0.05$. Note that the $\phi_{\max }$ boundary cannot be traced all the way, because the semi-analytic solutions of Simpson and Spiegelman (2011) only converge for $\lambda \leq 8.75$, for $(n, m)=(2,1)$. It is therefore possible that very large very slow waves exist that are not captured here.

The velocity of a porosity wave increases with size as, $\Lambda \sim \Delta^{2}$, because the segregation velocity of the melt increases with the compaction length as, $w_{0} \sim \delta_{0}^{2}$. Thus, the slope of the band in logarithmic $\Delta \Lambda$-space is two and the speed of a porosity wave increases rapidly with its size. Figure $11 a$ also shows the dependence of wave speed and size on the model parameters $\phi_{0}$ and $\lambda$. At constant $\phi_{0}$, an increase in $\lambda$ initially increases the size of the wave more rapidly than its velocity, see also Figure B.12. However, due to the limited range of the phase speed, $3 \geq \lambda \geq 8.75$, the dominant control on both size and velocity of the wave is the background porosity, $\phi_{0}$.

Unfortunately, $\phi_{0}$ is poorly constrained and often treated as an adjustable parameter (McKenzie, 1985b; Connolly, 1997). Figure 11a shows that decreasing $\phi_{0}$ will reduce the size of the porosity wave, but only at the expense of its velocity. Similarly, the wave velocity can be increased by elevating the background porosity. However, the maximum wave velocity that can be attained is limited by the small porosity approximation.

Numerical simulations of fluid flow in ductile rocks commonly lead to porosity waves that exceed 5\% porosity (Connolly and Podladchikov, 2000, Appold and Nunn, 2002, Connolly and Podladchikov, 2007, Šrámek et al., 2012; Joshi et al. 2012). These porosity waves are not described by the small porosity analysis presented here. However, such waves likely also transport mass in higher dimensions, as long as the porosity contrast to the background is sufficient to focus fluid flow into the wave.

Figure $11 b$ shows that the contours of the critical distribution coefficient, $D^{*}$, are mostly vertical. The ability of a porosity wave to transport tracers therefore increases with its size. This is due to the improved melt focusing in large high-amplitude waves. The behavior changes only in the vicinity of the $\lambda=3$ cut-off, where the contours become near horizontal, suggesting that 


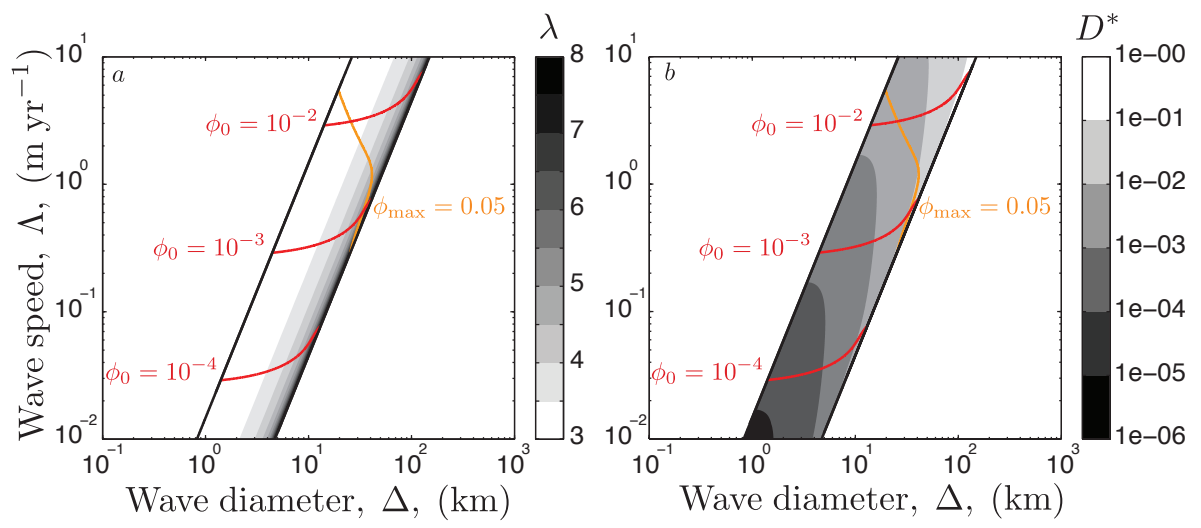

Figure 11: Band of possible solitary porosity waves given the dimensional length of the wave, $\Delta$, and the dimensional speed of the wave, $\Lambda$. The parameters used to generate this figure are typical upper-mantle values and can be found along with details of calculation in Appendix B Red contours show the background porosity, $\phi_{0}$ required for a wave given its dimension and speed. The gold contour represents, the chosen boundary of the small porosity approximation, $\phi_{\max }=0.05$. Analysis assuming small porosity applies to solutions below and to the left of this contour. a) Gray scale contours show phase speed, $\lambda$, given the diameter of the recirculating region, $\Delta$, and dimensional wave speed $\Lambda$. b) Gray scale contours illustrate the critical distribution coefficient, $D^{*}$, for tracers in solitary porosity waves.

transport improves with increasing velocity, and hence background porosity, $\phi_{0}$. Note that even waves with $\lambda=3$ can transport tracers, though the transported volume/area is very small, see Figure B.12.

The analysis in this manuscript is limited to the standard viscous rheology, with the constitutive exponents $(n, m)=(2,1)$, in two dimensions. The size and velocity of small amplitude porosity waves and their ability to transport tracers is likely to change dramatically with the choice of constitutive exponents and the spatial-dimension (Simpson and Spiegelman, 2011). In general, tracer transport is determined by the efficiency of fluid focusing for a particular wave (Figure $4 b$ ). This focusing is likely to be more efficient in three dimensions, so that the magnitude of $D^{*}$ in Figure $11 b$ should provide a lower limit. 


\section{Conclusions}

Here we show that higher-dimensional solitary porosity waves transport mass, because they focus the background fluid flow. This allows the fluid velocity to exceed the phase velocity in the high porosity center of the wave. Streamlines in a Lagrangian reference frame, moving with the phase velocity of the solitary porosity wave, show that the fluid recirculates in the core of the porosity wave. Mass within the recirculating region is transported by the porosity wave, because it is separated from the outer flow field by a circular, dividing streamline. Incompatible tracers are transported in the volume of the porosity wave enclosed by the dividing streamline of the fluid flow field. For compatible tracers, the radius of this circular dividing streamline, and hence the volume transported, decreases as the tracers becomes more compatible, until the transport ceases as the distribution coefficient exceeds a critical value. Unlike one-dimensional chromatography, transport in porosity waves does not produce chromatographic separations between relatively incompatible elements. Instead, it transports them together with the phase velocity of the porosity wave, and modifies their relative abundances. Therefore, porosity waves in ductile rocks provide a potential mechanism for the transport and preservation of geochemical signatures derived from melting of fertile heterogeneities in the mantle and the devolatilization metamorphic rocks. Sufficiently incompatible trace elements will travel together in an isolated batch of churning fluid. Furthermore, porosity waves provide a natural mechanism for mixing fluids and their geochemical signatures.

\section{Acknowledgments}

This work was supported by NSF Grant No. EAR 1301621. The authors would like to thank the Isaac Newton Institute for Mathematical Sciences for its hospitality during the programme "Melt in the Mantle" which was supported by EPSRC Grant No. EP/K032208/1. J.F.R. thanks the Leverhulme Trust for support. J.S.J would like to thank David Bercovici for facilitating travel to 
450

Cambridge, UK through NSF Grant No. EAR 1619535 to Yale University, in support of the Newton Institute programme on Melt in the Mantle. J.S.J and M.A.H. would like to thank John Lassiter for helpful discussions.

\section{Appendix A. Model equations}

\section{Appendix A.1. Dimensional model equations}

The equations governing the percolative flow of a fluid through a viscously deformable, permeable matrix were originally presented by McKenzie (1984), Scott and Stevenson (1984) and Fowler (1985b) and are given by

$$
\begin{gathered}
\frac{\partial \rho_{\mathrm{f}} \phi}{\partial t}+\nabla \cdot\left[\rho_{\mathrm{f}} \phi \mathbf{v}_{\mathrm{f}}\right]=\Gamma, \\
\frac{\partial \rho_{\mathrm{s}}(1-\phi)}{\partial t}+\nabla \cdot\left[\rho_{\mathrm{s}}(1-\phi) \mathbf{v}_{\mathrm{s}}\right]=-\Gamma, \\
\phi\left(\mathbf{v}_{\mathrm{f}}-\mathbf{v}_{\mathrm{s}}\right)=-\frac{K_{\phi}}{\mu}\left[\nabla P+\rho_{\mathrm{f}} g \hat{\mathbf{z}}\right], \\
\nabla P=\nabla \cdot\left(\eta\left[\nabla \mathbf{v}_{\mathrm{s}}+\nabla \mathbf{v}_{\mathrm{s}}^{T}-\frac{2}{3}\left(\nabla \cdot \mathbf{v}_{\mathrm{s}}\right) \mathbf{I}\right]\right)+\nabla\left(\zeta \nabla \cdot \mathbf{v}_{\mathrm{s}}\right)-\bar{\rho} g \hat{\mathbf{z}},
\end{gathered}
$$

where $\phi$ is the porosity or fluid fraction, $\rho_{\mathrm{f}}$ is the density of fluid, $\mu$ is the viscosity of the fluid, $\mathbf{v}_{\mathbf{f}}$ is the fluid velocity and $\Gamma$ is the fluid production rate. The density of the solid matrix is $\rho_{\mathrm{s}}$, its velocity is $\mathbf{v}_{\mathrm{s}}$ and its permeability is $K_{\phi}$. The densities of the fluid and solid are assumed to be constant, but not necessarily equal, so $\bar{\rho}=\rho_{\mathrm{f}} \phi+\rho_{\mathrm{s}}(1-\phi)$. Here $P$ is the pressure of the fluid, $\eta$ and $\zeta$ are the effective shear and bulk viscosities of the two phase mixture, $g$ is acceleration due to gravity, $\mathrm{z}$ is the vertical coordinate and $\hat{\mathbf{z}}=\nabla \mathrm{z}$ the upward pointing unit vector. For closure, constitutive relationships are needed for permeability and effective viscosity and a mass transfer rate, $\Gamma$, is required. For a full thermodynamic description of fluid production rate, melting-freezing, or dissolution-precipitation, additional conservation energy, material compositions and equations of state for reactions and phase equilibria are required (e.g. Rudge et al. (2011)).

If the shear viscosity $\eta$ is constant the momentum balance of the solid can 
be written as

$$
\nabla P=\eta \nabla \times \nabla \times \mathbf{v}_{\mathbf{s}}+\nabla\left[\left(\zeta+\frac{4}{3} \eta\right)\left(\nabla \cdot \mathbf{v}_{\mathbf{s}}\right)\right]-\bar{\rho} g \hat{\mathbf{z}},
$$

which allows the identification of three different contributions to the fluid pressure gradient,

$$
\nabla P=\nabla P^{*}+\nabla \mathcal{P}+\nabla P_{l}
$$

where $P^{*}$ is dynamic pressure, $P_{l}$ is lithostatic pressure in the absence of fluid $\left(P_{l} \equiv-\rho_{s} g z\right)$, and $\mathcal{P}$ is an effective compaction pressure defined by

$$
\mathcal{P} \equiv \xi_{\phi} \nabla \cdot \mathbf{v}_{\mathrm{s}}
$$

where $\xi_{\phi} \equiv \zeta+\frac{4}{3} \eta$. Substituting A.3 into the system A.1 yields

$$
\begin{gathered}
\frac{\partial \phi}{\partial t}+\mathbf{v}_{\mathbf{s}} \cdot \nabla \phi=(1-\phi) \frac{\mathcal{P}}{\xi_{\phi}}+\frac{\Gamma}{\rho_{\mathrm{s}}}, \\
-\nabla \cdot \frac{K_{\phi}}{\mu} \nabla \mathcal{P}+\frac{\mathcal{P}}{\xi_{\phi}}=\nabla \cdot \frac{K_{\phi}}{\mu}\left(\nabla P^{*}-\Delta \rho g \hat{\mathbf{z}}\right)+\Gamma \frac{\Delta \rho}{\rho_{\mathrm{f}} \rho_{\mathrm{s}}}, \\
\nabla \cdot \mathbf{v}_{\mathrm{s}}=\frac{\mathcal{P}}{\xi_{\phi}}, \\
\nabla P^{*}=\eta \nabla \times \nabla \times \mathbf{v}_{\mathbf{s}}+\phi \Delta \rho g \hat{\mathbf{z}},
\end{gathered}
$$

where $\Delta \rho=\rho_{\mathrm{s}}-\rho_{\mathrm{f}}$. Equation A.5b is a modified Helmholtz equation for compaction pressure $\mathcal{P}$ that reduces to the familiar Darcy's law in the limit of large $\xi_{\phi}$. Equation A.5c relates the divergence of the solid flow field to the compaction pressure and the resistance of the media to volumetric expansion and contraction. Finally, Equation A.5d is a Stokes-like equation for solid velocity and dynamic pressure driven by deviatoric stresses with buoyancy driven by porosity. Equation A.5c can be decoupled from Equation A.5d by applying a Helmholtz decomposition to the solid velocity field, $\mathbf{v}_{\mathrm{s}}=-\nabla U+\nabla \times \mathbf{\Psi}$, where $U$ is the scalar potential and $\Psi$ is the vector potential Spiegelman, 1993c). Lastly, using (A.3), the fluid flux relative to the movement of the solid matrix is given by

$$
\mathbf{q}_{r}=\phi\left(\mathbf{v}_{\mathrm{f}}-\mathbf{v}_{\mathrm{s}}\right)=-\frac{K_{\phi}}{\mu}\left(\nabla\left[P^{*}+\mathcal{P}-\Delta \rho g \mathrm{z}\right]\right) .
$$


For a tracer that partitions into both phases the bulk concentration in the system is conserved and given by,

$$
C=\phi \rho_{\mathrm{f}} x_{\mathrm{f}}+(1-\phi) \rho_{\mathrm{s}} x_{\mathrm{s}}
$$

where $\rho_{\mathrm{p}}$ and $x_{\mathrm{p}}$ are the densities and mass fractions of tracer partitioned across the solid phases and fluid phase respectively. At local chemical equilibrium the partition coefficient, $D$ defined in (6), can be used to eliminate $x_{\mathrm{s}}$ from (A.7), so that

$$
C=(\phi+(1-\phi) D) \rho_{\mathrm{f}} x_{\mathrm{f}}
$$

Tracer is transported by advection of the two phases, molecular diffusion and mechanical dispersion. The latter two are usually negligible on transport distances considered in melt migration. Therefore, we focus on advective transport here, so that the total mass conservation equation is given by

$$
\frac{\partial C}{\partial t}+\nabla \cdot\left[\left(\phi \mathbf{v}_{\mathrm{f}}+(1-\phi) \mathbf{v}_{\mathrm{s}} D\right) \rho_{\mathrm{f}} x_{\mathrm{f}}\right]=0
$$

where $\mathbf{v}_{\mathrm{f}}$ and $\mathbf{v}_{\mathrm{s}}$ are the fluid and solid velocities. Using A.8 to eliminate $\rho_{\mathrm{f}} x_{\mathrm{f}}$ the evolution equation for the bulk composition is simply

$$
\frac{\partial C}{\partial t}+\nabla \cdot\left[\mathbf{v}_{\mathrm{e}} C\right]=0,
$$

where the effective velocity of the tracer is given by

$$
\mathbf{v}_{\mathrm{e}}=\frac{\phi \mathbf{v}_{\mathrm{f}}+(1-\phi) \mathbf{v}_{\mathrm{s}} D}{\phi+(1-\phi) D}
$$

\section{Appendix A.3. Scaling}

The compaction length is the intrinsic length scale for the system of governing equations given by A.5. The compaction length is the solid phase relaxation distance for a piezometric overpressure dilating the porosity, or the length scale over which $\mathcal{P}$ responds to variations in the relative fluid flux $\mathbf{q}_{r}$. 
Using a reference porosity, $0<\phi_{0}<1$, the characteristic compaction length, is given by,

$$
\delta_{0}=\sqrt{\frac{K_{0} \xi_{\phi_{0}}}{\mu}},
$$

where $K_{0}=K_{\phi}\left(\phi_{0}\right)$ and $\xi_{\phi_{0}}=\xi_{\phi}\left(\phi_{0}\right)$ in equation (2). The buoyancy-driven separation flux of the fluid relative to the solid is given by

$$
\phi_{0} w_{0}=\frac{K_{0} \Delta \rho g}{\mu}
$$

where $w_{0}$ is the characteristic fluid segregation velocity. Using (A.12) and A.13) along with material properties, the suite of model equations can be scaled by the following,

$$
\begin{array}{rl}
\mathbf{x}=\delta_{0} \mathbf{x}^{\prime} & \nabla=\nabla^{\prime} / \delta_{0} \\
\phi=\phi_{0} \phi^{\prime} & t=\left(\delta_{0} / w_{0}\right) t^{\prime} \\
\mathbf{v}_{\mathrm{f}}=w_{0} \mathbf{v}_{\mathrm{f}}^{\prime} & \mathbf{v}_{\mathrm{s}}=\phi_{0} w_{0} \mathbf{v}_{\mathrm{s}}^{\prime} \\
\mathcal{P}=\Delta \rho g \delta_{0} \mathcal{P}^{\prime} & P^{*}=\phi_{0} \Delta \rho g \delta_{0} P^{* \prime} \\
K_{\phi}=K_{0} K_{\phi}^{\prime} & \xi_{\phi}=\frac{\eta}{\phi_{0}} \xi_{\phi}^{\prime} \\
C=\phi_{0} \rho_{\mathrm{f}} \mathcal{C} & U=\phi_{0} w_{0} \delta_{0} \mathcal{U} \\
\boldsymbol{\Psi}=\phi_{0} w_{0} \delta_{0} \boldsymbol{\Psi}^{\prime} & \Gamma=\frac{\rho_{\mathrm{s}} \phi_{0} w_{0}}{\delta_{0}} \Gamma^{\prime}
\end{array}
$$

where primes denote dimensionless variables. Substituting these scales into the system of equations given by A.1 and dropping the primes we obtain the dimensionless system of governing equations

$$
\begin{gathered}
\frac{\partial \phi}{\partial t}+\phi_{0} \mathbf{v}_{\mathrm{s}} \cdot \nabla \phi=\left(1-\phi_{0} \phi\right) \frac{\mathcal{P}}{\xi_{\phi}}+\Gamma, \\
-\nabla \cdot\left[K_{\phi} \nabla \mathcal{P}\right]+\frac{\mathcal{P}}{\xi_{\phi}}=\nabla \cdot\left[K_{\phi}\left(\phi_{0} \nabla P^{*}-\hat{\mathbf{z}}\right)\right]+\Gamma \frac{\Delta \rho}{\rho_{\mathrm{f}}}, \\
-\nabla^{2} \mathcal{U}=\frac{\mathcal{P}}{\xi_{\phi}}, \\
\nabla P^{*}=\nabla \times \nabla \times \nabla \times \boldsymbol{\Psi}+\phi \hat{\mathbf{z}} .
\end{gathered}
$$


The volumetric flux of the fluid is given by

$$
\mathbf{q}_{r}=\phi\left(\mathbf{v}_{\mathrm{f}}-\phi_{0} \mathbf{v}_{\mathbf{s}}\right)=-K\left(\nabla \mathcal{P}+\phi_{0} \nabla P^{*}-\hat{\mathbf{z}}\right) .
$$

Substituting A.14 into A.8 A.11, the scaled dimensionless tracer evolution equations is

$$
\frac{\partial \mathcal{C}}{\partial t}+\nabla \cdot\left[\mathbf{v}_{\mathrm{e}} \mathcal{C}\right]=0
$$

where the dimensionless bulk composition and effective velocity are given by

$$
\mathcal{C}=\left(\phi+\left(1-\phi_{0} \phi\right) D / \phi_{0}\right) x_{\mathrm{f}}
$$

and

$$
\mathbf{v}_{\mathrm{e}}=\frac{\phi \mathbf{v}_{\mathrm{f}}+\left(1-\phi_{0} \phi\right) \mathbf{v}_{\mathrm{s}} D}{\phi+\left(1-\phi_{0} \phi\right) D / \phi_{0}} .
$$

Here we have dropped the primes indicating dimensionless variables.

Appendix A.4. Small porosity approximation and the reduced model for fluid migration

Throughout this manuscript we apply the small porosity approximation, assuming that the ambient mantle has a porosity $\phi_{0} \ll 1$. Application of the small porosity limit to the dimensionless system of governing equations A.15 results in the following simplifications: The solid volume fraction is unity, $(1-$ $\left.\phi_{0} \phi\right) \approx 1$. Equation A.15d decouples, because terms containing $P^{*}$ in other equations are negligible. Terms containing $\mathbf{v}_{s}$ are negligible, except the term containing $D$ in A.19. After the application of these simplifications to the dimensionless system A.15, the system reduces to

$$
\begin{aligned}
\frac{\partial \phi}{\partial t} & =\frac{\mathcal{P}}{\xi_{\phi}}, \\
-\nabla \cdot K_{\phi} \nabla \mathcal{P}+\frac{\mathcal{P}}{\xi_{\phi}} & =-\nabla \cdot K_{\phi} \hat{\mathbf{z}}, \\
-\nabla^{2} \mathcal{U} & =\frac{\mathcal{P}}{\xi_{\phi}} .
\end{aligned}
$$


Using the scaled relationship for permeability, $K_{\phi}=\phi^{n}$, the phase velocities are given by

$$
\mathbf{v}_{\mathrm{f}}=-\phi^{(n-1)}(\nabla \mathcal{P}-\hat{\mathbf{z}}) \quad \text { and } \quad \mathbf{v}_{s}=-\nabla \mathcal{U}
$$

The evolution of the dimensionless bulk composition is given by

$$
\frac{\partial \mathcal{C}}{\partial t}+\nabla \cdot\left[\frac{\phi \mathbf{v}_{\mathrm{f}}+\mathbf{v}_{\mathrm{s}} D}{\phi+D / \phi_{0}} \mathcal{C}\right]=0 .
$$

\section{Appendix B. Dimensional solitary porosity waves}

To explore the relevancy of solitary porosity waves as a transport mechanism in regional metamorphic fluid release and magma transport applications alike, the wavelength or size of the wave and speed of the wave must be known. Here we define the size of a solitary porosity wave to be the diameter of the circular dividing streamline, $\Delta=\mathcal{D}(\lambda) \delta_{0}$, where $\mathcal{D}(\lambda)$ for $\lambda \in[3,8]$ is an empirical fit to the semi-analytic solutions shown in Figure $8 c$ and further illustrated in Figure B.12. The dimensional speed of the wave, $\Lambda=\lambda w_{0}$ is simply the phase speed of the wave multiplied by the characteristic segregation velocity due to the buoyancy of the melt. Determining the physical size and speed of porosity waves is complicated by three factors:

1. The strong dependence of the solitary waves on the constitutive exponents $n$ and $m$ as well as the physical dimension.

2. The natural variation and the uncertainty in the magnitude of the physical parameters (e.g. grain size of the ambient mantle background).

3. The presence of the two parameters $\phi_{0}$ and $\lambda$ that are often unconstrained and hence commonly used as fitting parameters.

All results presented in Sections 2 and 3 are for porosity waves with constitutive exponents $(n, m)=(2,1)$ and in two-dimensions. Therefore, the discussion of the effect of these parameters is beyond the scope of this manuscript, but clearly an important question for future work. Similarly, we will not explore the 

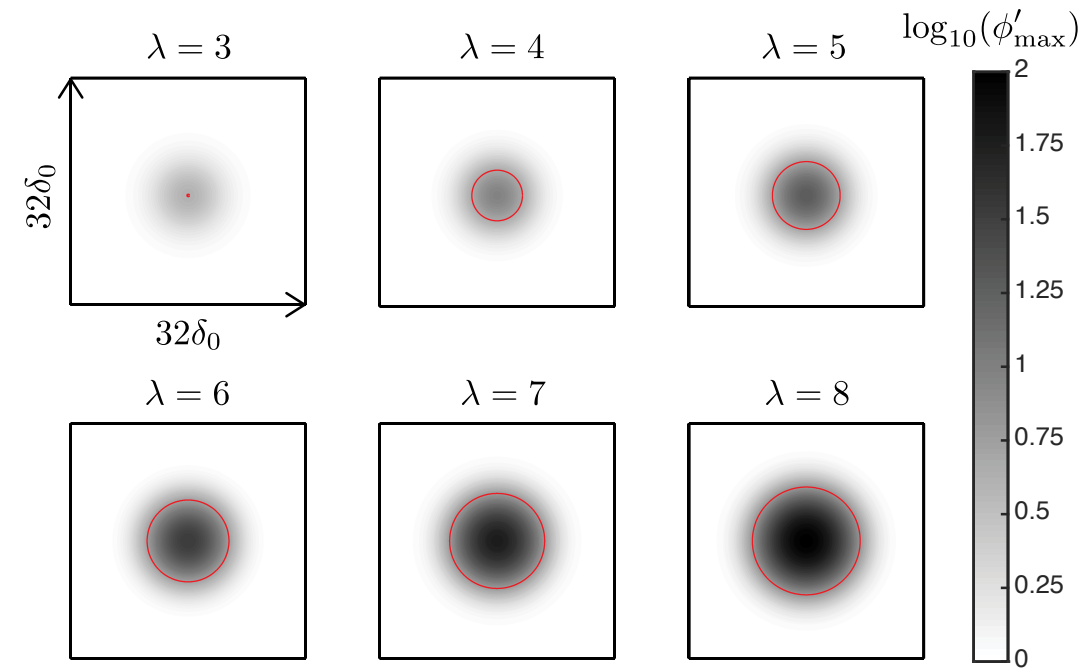

Figure B.12: Gray scale contours show the logarithm of scaled porosity field, $\phi^{\prime}=\phi / \phi_{0}$. The maroon contour shows the circular, dividing streamline, the diameter of which is $\mathcal{D}(\lambda)$. It is worth noting that the dividing streamline and amplitude increase considerably with phase speed $\lambda$, the extent of the porosity anomaly grows much more slowly. This illustrates that relatively fast moving waves are higher amplitude and thus focus melt far more efficiently. 

$\phi_{0}$ and $\lambda$, which are often unclear.

Table B.1: Parameters required for Equation B.7)

\begin{tabular}{cccc}
\hline \hline Variable & Description & Value & Dimensions \\
\hline$d$ & Grain size & $10^{-3}$ & $\mathrm{~m}$ \\
$g$ & Gravity & 9.81 & $\mathrm{~m} \mathrm{~s}^{-2}$ \\
$\eta$ & Shear viscosity of solid & $10^{19}$ & $\mathrm{~Pa} \mathrm{~s}$ \\
$\tau$ & Dimensionless parameter in $K_{0}$ & 1600 & - \\
$\mu$ & Viscosity of fluid & 1 & $\mathrm{~Pa} \mathrm{~s}$ \\
$\Delta \rho$ & Density difference of melt \& matrix & 500 & $\mathrm{~kg} \mathrm{~m}^{-3}$ \\
\hline
\end{tabular}

The relevant physical relationships for solitary porosity waves form a nonlinear system of algebraic equations,

$$
\begin{array}{r}
\Lambda=\lambda w_{0}, \\
\Delta=\mathcal{D}(\lambda) \delta_{0}, \\
w_{0}=\frac{K_{0} \Delta \rho g}{\phi_{0} \mu}, \\
K_{0}=\frac{d^{2} \phi_{0}^{2}}{\tau}, \\
\delta_{0}=\sqrt{\frac{K_{0} \xi_{0}}{\mu},} \\
\xi_{0}=\zeta_{0}+\frac{4}{3} \eta,
\end{array}
$$

where $\mathcal{D}(\lambda)$ is a cubic fit of model output as shown in $8 c$ and Table B.2, and $\zeta_{0}=\zeta^{*} \phi^{*} \eta / \phi_{0}$. The ratio of bulk to shear viscosity of the matrix at reference porosity, $\phi^{*}$, is denoted, $\zeta^{*}$, and may range from $10-200$. The product of $\zeta^{*} \phi^{*}$ has been estimated both experimentally and theoretically ranging from $1-10$ Cooper, 1990, Hewitt and Fowler, 2008). Here we choose $\zeta^{*} \phi^{*}=1$ so equation B.6 becomes $\xi_{0}=\eta\left(1 / \phi_{0}+4 / 3\right)$. Lastly, $\tau$ (a dimensionless parameter in the permeability, $\left.K_{0}\right)$ is chosen to be 1600 , which is appropriate for $n=2($ Frank 
1968; von Bargen and Waff, 1986, Cheadle, 1989).

\begin{tabular}{c|ccccc}
\multicolumn{5}{c}{ Table B.2: Polynomial fit for $f(\lambda)=a_{0}+a_{1} \cdot \lambda+a_{2} \cdot \lambda^{2}+a_{3} \cdot \lambda^{3}+a_{4} \cdot \lambda^{4}$} \\
\hline \hline & $a_{0}$ & $a_{1}$ & $a_{2}$ & $a_{3}$ & $a_{4}$ \\
\hline \hline $\mathcal{D}$ & -32.1647 & 17.4541 & -2.4443 & 0.1237 & 0 \\
$\phi_{\max }^{\prime}$ & -97.6775 & 66.7686 & -14.9377 & 1.2758 & 0 \\
$D^{*}$ & 31.8696 & -28.2758 & 8.8501 & -1.1785 & 0.0654 \\
\hline
\end{tabular}

The nonlinear system of algebraic equations $B .1-B .6$ are combined to obtain a single residual function,

$$
R(\Delta, \Lambda, \lambda)=\Delta-\mathcal{D}(\lambda) \frac{1}{\lambda}\left(\frac{\Lambda \tau \mu}{d \Delta \rho g}\right) \sqrt{\frac{\eta}{\tau \mu}\left[\frac{4}{3}+\left(\lambda \frac{d^{2} \Delta \rho g}{\Lambda \tau \mu}\right)\right]}=0
$$

Given values for $\Lambda$ and $\Delta$ the residual function is solved for $\lambda$ and the relationships described in B.1 B.6 are determined. Additionally, the background porosity can be expressed by rearranging (B.1) as,

$$
\phi_{0}=\frac{1}{\lambda} \frac{\Lambda \tau \mu}{d^{2} \Delta \rho g} \text {. }
$$

This background porosity is contoured in Figure 11. General contours for porosity maximum porosity, or amplitude plus the background porosity, $\phi_{\max }^{\prime}=A+1$, are obtained using a cubic fit with coefficients provided in Table B.2. The dimensional gold contours for $\phi=0.05$ in Figure $11 a$ are calculated by multiplying contours obtained from this cubic fit by the background porosity. The critical distribution coefficient, $D^{*}$, contoured in Figure $11 b$ is fit using a quartic polynomial to the model data plotted in figure $8 b$ with $D^{*} / \phi_{0}$. Coefficients for this fit are also given in Table B.2. Gray-scale contours for $D^{*}$ in Figure $11 b$ are also dimensionalized by multiplying by the background porosity.

Appold, M., Nunn, J., 2002. Numerical models of petroleum migration via buoyancy-driven porosity waves in viscously deformable sediments. Geofluids 2, 233-247. doi:10.1046/j.1468-8123.2002.00040.x. 
Bailey, R., 1990. Trapping of aqueous fluids in the deep crust. Geophysical Research Letters 17, 1129-1132. doi:10.1029/GL017i008p01129

Barcilon, V., Lovera, O., 1989. Solitary waves in magma dynamics. Journal of Fluid Mechanics 204, 121-133. doi:10.1017/S0022112089001680.

Barcilon, V., Richter, F., 1986. Nonlinear waves in compacting media. Journal of Fluid Mechanics 164, 429-448.

von Bargen, N., Waff, H., 1986. Permeabilities, interfacial areas and curvatures of partially molten systems: Results of numerical computations of equilibrium microstructures. Journal of Geophysical Research 91, 9261. URL: http://doi.wiley.com/10.1029/JB091iB09p09261, doi 10 . 1029/JB091iB09p09261.

Baumgartner, L.P., Rumble III, D., 1988. Transport of stable isotopes: I. Develomment of a kinetic continuum theory for stable isotope transport. Contributions to Mineralogy and Petrology 98, 417-430. doi 10.1007/BF00372362.

Bickle, M.J., McKenzie, D., 1987. The transport of heat and matter by fluids during metamorphism. Contributions to Mineralogy and Petrology 95, 384392. doi $10.1007 / \mathrm{BF} 00371852$.

Bowman, J.R., Willett, S.D., 1991. Spatial patterns of oxygen isotope exchange during one-dimensional fluid infiltration. Geophysical Research Letters 18, 971-974.

Cheadle, M., 1989. Properties of texturally equilibrated two-phase aggregates. Ph.D. thesis. University of Cambridge.

Connolly, J., 2010. The mechanics of metamorphic fluid expulsion. Elements 6, 165-172. doi $10.2113 /$ gselements.6.3.165.

Connolly, J., Podladchikov, Y., 2000. Temperature-dependent viscoelastic compaction and compartmentalization in sedimentary basins. Tectonophysics 324 , 137-168. doi:10.1016/S0040-1951(00)00084-6. 
Connolly, J., Podladchikov, Y., 2007. Decompaction weakening and channeling instability in ductile porous media: Implications for asthenospheric melt segregation. Journal of Geophysical Research 112, B10205. URL: http: //doi.wiley.com/10.1029/2005JB004213, doi:10.1029/2005JB004213.

Connolly, J.A.D., 1997. Devolatilization-generated fluid pressure and deformation-propagated fluid flow during prograde regional metamorphism.

Journal of Geophysical Research-Solid Earth 102, 18149-18173. doi 10.1029/ $97 \mathrm{jb} 00731$.

Connolly, J.A.D., Podladchikov, Y.Y., 2015. An analytical solution for solitary porosity waves: Dynamic permeability and fluidization of nonlinear viscous and viscoplastic rock. Geofluids 15, 269-292. doi:10.1111/gfl.12110.

Cooper, R.F., 1990. Differential stress-induced melt migration: an experimental approach. Journal of Geophysical Research B 95, 6979-6992.

De Paolo, J., 1996. High-frequency isotopic variations in the Mauna Kea tholeiitic basalt sequence: Melt zone dispersivity and chromatography. Journal of Geophysical Research 101, 11855-11864.

Fowler, A., 1985a. A mathematical model of magma transport in the asthenosphere. Geophysical \& Astrophysical Fluid Dynamics 33, 63-96. doi 10 . 1080/03091928508245423.

Fowler, A., 1985b. A mathematical model of magma transport in the asthenosphere. Geophys Astrophys Fluid Dyn 33, 63-96. doi 10.1080/ 03091928508245423 .

Frank, F., 1968. Two-component flow model for convection in the Earth's up¿ per mantle. Nature 220, 350-352. URL: http://adsabs.harvard.edu/abs/ 1968Natur.220 . .350F, doi:10.1038/220350a0.

Ghanbarzadeh, S., Prodanovic, M., Hesse, M., 2014. Percolation and grain boundary wetting in anisotropic texturally equilibrated pore networks. Physical Review Letters 113, 1-5. 
Hauri, E.H., 1997. Melt migration and mantle chromatography , 1: simplified theory and conditions for chemical and isotopic decoupling. Earth and Planetary Science Letters 153, 1-19.

Hauri, E.H., Kurz, M.D., 1997. Melt migration and mantle chromatography, 2: a time-series Os isotope study of Mauna Loa volcano, Hawaii. Earth and Planetary Science Letters 153, 21-36. doi:10.1016/S0012-821X(97)00158-1

Hewitt, I., Fowler, A., 2008. Partial melting in an upwelling mantle column. Proceedings of the Royal Society A: Mathematical, Physical and Engineering Science 464, 2467-2491. doi:10.1098/rspa.2008.0045.

Hofmann, A., 1972. Chromatographic theory of infiltration metasomatism and its application to feldspars. American Journal of Science 272, 69-90.

Joshi, A., Appold, M., 2016. Potential of porosity waves for methane transport in the Eugene Island field of the Gulf of Mexico basin. Marine and Petroleum

Geology 75, 1-13. URL: http://dx.doi.org/10.1016/j.marpetgeo.2016. 04.005 doi:10.1016/j.marpetgeo.2016.04.005.

Joshi, A., Appold, M., Nunn, J., 2012. Evaluation of solitary waves as a mechanism for oil transport in poroelastic media: A case study of the South

Eugene Island field, Gulf of Mexico basin. Marine and Petroleum Geology 37, 53-69. URL: http://dx.doi.org/10.1016/j.marpetgeo.2012.06.011, doi:10.1016/j.marpetgeo.2012.06.011.

Katz, R., 2008. Magma dynamics with the enthalpy method: Benchmark solutions and magmatic focusing at mid-ocean ridges. Journal of Petrology 49, 2099-2121. doi:10.1093/petrology/egn058.

Katz, R.F., Rudge, J.F., 2011. The energetics of melting fertile heterogeneities within the depleted mantle. Geochemistry, Geophysics, Geosystems 12, 1-22. doi:10.1029/2011GC003834.

Korzhinskii, D.S., 1965. The theory of Systems with Perfectly Mobile Components and Processes of Mineral Formation. 
Liang, Y., 2008. Simple models for dynamic melting in an upwelling heterogeneous mantle column: Analytical solutions. Geochimica et Cosmochimica Acta 72, 3804-3821. URL: http://linkinghub.elsevier.com/retrieve/ pii/S0016703708003311, doi:10.1016/j.gca.2008.05.050

McKenzie, D., 1984. The Generation and Compaction of Partially Molten Rock. Journal of Petrology 25, 713-765.

McKenzie, D., 1985a. 230Th-238U disequilibrium and the melting processes beneath ridge axes. Earth and Planetary Science Letters 72, 149-157. doi 10. 1016/0012-821X(85)90001-9.

McKenzie, D., 1985b. The extraction of magma from the crust and mantle. Earth and Planetary Science Letters 74, 81-91. doi 10.1016/0012-821X(85) 90168-2.

McKenzie, D., 1987. The compaction of igneous and sedimentary rocks. Journal of the Geological Society 144, 299-307. URL: http:// jgs.geoscienceworld.org/content/144/2/299, doi 10.1144/gsjgs.144. 2.0299 .

McSween, H., Richardson, S., Uhle, M., 2003. Geochemistry. 2nd ed., Columbia University Press.

Miller, K.J., Zhu, W.l., Montési, L.G.J., Gaetani, G.A., 2014. Experimental quantification of permeability of partially molten mantle rock. Earth and Planetary Science Letters 388, 273-282. URL: http://dx.doi.org/10. 1016/j.epsl.2013.12.003, doi 10.1016/j.epsl.2013.12.003.

Navon, O., Stolper, E., 1987. Geochemical consequences of melt percolation: the upper mantle as a chromatographic column. The Journal of Geology 95, 285-307.

Norton, D., Taylor, H.P., 1979. Quantitative simulation of the hydrothermal systems of crystallizing magmas on the basis of transport theory and oxygen 
isotope data: An analysis of the skaergaard intrusion. Journal of Petrology 20, 421-486. doi $10.1093 /$ petrology/20.3.421.

Phipps Morgan, J., 1987. Melt migration beneath mid-ocean spreading centers. Geophysical Research Letters 14, 1238-1241. doi 10.1029/GL014i012p01238

Richter, F., McKenzie, D., 1984. Dynamical Models for Melt Segregation from a Deformable Matrix. The Journal of Geology 92, 729-740.

Richter, F.M., Daly, S.F., 1989. Dynamical and chemical effects of melting a heterogeneous source. Journal of Geophysical Research v. 94, 499-12. doi 10. 1029/JB094iB09p12499.

Rudge, J.F., Bercovici, D., Spiegelman, M., 2011. Disequilibrium melting of a two phase multicomponent mantle. Geophysical Journal International 184, 699-718. doi:10.1111/j.1365-246X.2010.04870.x.

Scott, D., Stevenson, D., 1984. Magma solitons. Geophysical Research Letters 11, 1161-1164. URL: http://onlinelibrary.wiley.com/doi/10.1029/ GL011i011p01161/full.

Scott, D., Stevenson, D., 1986. Magma ascent by porous flow. Journal of Geophysical Research B 91, 9283. doi 10.1029/JB091iB09p09283

Simpson, G., Spiegelman, M., 2011. Solitary wave benchmarks in magma dynamics. Journal of Scientific Computing 49, 268-290. doi 10.1007/ s10915-011-9461-y.

Skarbek, R., Rempel, A., 2016. Dehydration-induced porosity waves and episodic tremor and slip. Geochemistry Geophysics Geosystems 17, 28252834. doi $10.1002 / 2016 \mathrm{GC006406}$.

Solano, J.M.S., Jackson, M.D., Sparks, R.S.J., Blundy, J., 2014. Evolution of major and trace element composition during melt migration through crystalline mush: Implications for chemical differentiation in the crust. American 
Journal of Science 314, 895-939. URL: http://www.ajsonline.org/cgi/ doi/10.2475/05.2014.01, doi $10.2475 / 05.2014 .01$.

Sparks, D.W., Parmentier, E., 1991. Melt extraction from the mantle beneath spreading centers. Earth and Planetary Science Letters 105, 368-377. doi:10. 1016/0012-821X(91) 90178-K.

Spiegelman, M., 1993a. Flow in deformable porous media. Part 1 Simple analysis. Journal of Fluid Mechanics 247, 17-38. doi 10.1017/S0022112093000369

Spiegelman, M., 1993b. Flow in deformable porous media. Part 2 numerical analysis-the relationship between shock waves and solitary waves. Journal of Fluid Mechanics 247, 39-63. doi $10.1017 /$ S0022112093000370

Spiegelman, M., 1993c. Physics of melt extraction: theory, implications, applications. Philosophical Transactions of the Royal Society A: Mathematical, Physical and Engineering Sciences 342, 23-41.

Spiegelman, M., 1994. Geochemical Effects of Magmatic Solitary Waves .2. Some 1 Analysis. Geophysical Journal International 117, 296-300. URL: <GotoISI> : //A1994NJ00600003.

Šrámek, O., Milelli, L., Ricard, Y., Labrosse, S., 2012. Thermal evolution and differentiation of planetesimals and planetary embryos. Icarus

217, 339-354. URL: http://linkinghub.elsevier.com/retrieve/pii/ S0019103511004489, doi $10.1016 / j$. icarus.2011.11.021.

Thompson, A., Connolly, J., 1990. Metamorphic fluids and anomalous porosities in the lower crust. Tectonophysics 182, 47-55. URL: http: 1. //linkinghub.elsevier.com/retrieve/pii/0040195190903415, doi 10 . 1016/0040-1951(90)90341-5.

Tian, M., Ague, J., 2014. The impact of porosity waves on crustal reaction progress and CO2 mass transfer. Earth and Planetary Science Letters 390, 80-92. URL: http://linkinghub.elsevier.com/retrieve/pii/ S0012821X13007590 doi 10.1016/j.epsl.2013.12.044. 
Wark, D., Watson, E., 1998. Grain-scale permeabilities of texturally equilibrated, monomineralic rocks. Earth and Planetary Science Letters 164, 591-605. URL: http://www.sciencedirect.com/science/article/pii/ S0012821X98002520.

Watson, S., Spiegelman, M., 1994. Geochemical Effects of Magmatic Solitary Waves .I. Numerical results. Geophysical Journal International 117, 284-295.

Weatherley, S.M., Katz, R.F., 2012. Melting and channelized magmatic flow in chemically heterogeneous, upwelling mantle. Geochemistry Geophysics Geosystems 13, Q0AC18. URL: http://doi.wiley.com/10.1029/ 2011GC003989, doi:10.1029/2011GC003989.

White, W., 2013. Geochemistry. Wiley-Blackwell.

Whitehead, J., Helfrich, K., 1988. Wave transport of deep mantle material.

Nature 336, 59-61. URL: http://www.nature.com/nature/journal/v336/ n6194/abs/336059a0.html.

Wiggins, C., Spiegelman, M., 1995. Magma migration and magmatic solitary waves in 3-D. Geophysical Research Letters 22, 1289-

1292. URL: http://onlinelibrary.wiley.com/doi/10.1029/95GL00269/ full, doi $10.1029 / 95 \mathrm{GL} 00269$. 